\title{
MODULE CATEGORIES OVER AFFINE SUPERGROUP SCHEMES
}

\author{
SHLOMO GELAKI
}

\begin{abstract}
Let $k$ be an algebraically closed field of characteristic 0 or $p>2$. Let $\mathcal{G}$ be an affine supergroup scheme over $k$. We classify the indecomposable exact module categories over the tensor category $\mathrm{sCoh}_{\mathrm{f}}(\mathcal{G})$ of (coherent sheaves of) finite dimensional $\mathcal{O}(\mathcal{G})$ supermodules in terms of $(\mathcal{H}, \Psi)$-equivariant coherent sheaves on $\mathcal{G}$. We deduce from it the classification of indecomposable geometrical module categories over $\operatorname{sep}(\mathcal{G})$. When $\mathcal{G}$ is finite, this yields the classification of all indecomposable exact module categories over the finite tensor category $\operatorname{sRep}(\mathcal{G})$. In particular, we obtain a classification of twists for the supergroup algebra $k \mathcal{G}$ of a finite supergroup scheme $\mathcal{G}$, and then combine it with EG2, Corollary 4.1] to classify finite dimensional triangular Hopf algebras with the Chevalley property over $k$.
\end{abstract}

\section{Contents}

1. Introduction

2. Preliminaries

2.1. Affine supergroup schemes

2.2. Module categories over tensor categories.

3. The tensor category $\operatorname{sCoh}_{\mathrm{f}}(\mathcal{G})$

4. Equivariant quasi-coherent sheaves

5. Exact module categories over $\mathrm{sCoh}_{\mathrm{f}}(\mathcal{G})$

6. Exact module categories over $\operatorname{sRep}(\mathcal{G})$

6.1. Module categories.

6.2. Semisimple module categories of rank 1.

6.3. Exact module categories over finite supergroup schemes

7. The classification of triangular Hopf algebras with the Chevalley property

7.1. Twists for $k \mathcal{G}$.

7.2. Minimal twists for $k \mathcal{G}$.

Date: January 26, 2021.

Key words and phrases. affine supergroup scheme; tensor category; module category; twist; triangular Hopf algebra. 
7.3. Triangular Hopf algebras $\quad 23$

References $\quad 24$

\section{INTRODUCTION}

Let $k$ be an algebraically closed field of characteristic 0 or $p>2$. Let $G$ be a finite group scheme over $k$. Consider the finite tensor category $\operatorname{Coh}(G)$ of finite dimensional $\mathcal{O}(G)$-modules over $k$, and the finite tensor category $\operatorname{Rep}(G)$ of finite dimensional rational representations of $G$ over $k$. In G2 we classified the indecomposable exact module categories over $\operatorname{Rep}(G)$, generalizing the classification of Etingof and Ostrik [EO] for constant groups $G$. In particular, we obtained the classification of twists for the group algebra $k G$, reproducing the classification given by Movshev for constant groups $G$ in zero characteristic [Mo].

The goal of this paper is to extend G2 to the super case, and then combine it with [EG2, Corollary 4.1] to classify finite dimensional triangular Hopf algebras with the Chevalley property over $k$ (as promised in [EG2, Remark 1.5(3)]).

Let $\mathcal{G}$ be a finite supergroup scheme over $k$. Following [G2, we first classify the indecomposable exact module categories over $\operatorname{sep}(\mathcal{O}(\mathcal{G}))$, where $\mathcal{O}(\mathcal{G})$ is the coordinate Hopf superalgebra of $\mathcal{G}$, and then use the fact that they are in bijection with the indecomposable exact module categories over $\operatorname{sRep}(\mathcal{G})$ [EO to get the classification of the latter ones. The reason we approach it in this way is that $\operatorname{sRep}(\mathcal{O}(\mathcal{G}))$ is tensor equivalent to the tensor category $\operatorname{sCoh}_{\mathrm{f}}(\mathcal{G})=\mathrm{sCoh}(\mathcal{G})$ (of coherent sheaves) of finite dimensional $\mathcal{O}(\mathcal{G})$-supermodules with the tensor product of convolution of sheaves, which allows us to use geometric tools and arguments.

In fact, in Theorem 5.5 we classify the indecomposable exact module categories over $\operatorname{sCoh}_{\mathrm{f}}(\mathcal{G})$, where $\mathcal{G}$ is any affine supergroup scheme over $k$ (i.e., $\mathcal{G}$ is not necessarily finite). The classification is given in terms of certain $(\mathcal{H}, \Psi)$-equivariant coherent sheaves on $\mathcal{G}$ (see Definition 4.1). However when $\mathcal{G}$ is not finite, not all indecomposable exact module categories over $\operatorname{sep}(\mathcal{G})$ are obtained from those over $\operatorname{sCoh}_{\mathrm{f}}(\mathcal{G})$ (see Theorem 6.6 and Remark 6.7); we refer to those which are as geometrical. So the classification of exact module categories (even fiber functors) over $\operatorname{sep}(\mathcal{G})$ for infinite affine supergroup schemes $\mathcal{G}$ remains unknown (even when $\mathcal{G}$ is a linear algebraic group over $\mathbb{C}$, see [G2]).

As a consequence of our results, combined with [AEGN, EO], we obtain in Corollary 7.1 that gauge equivalence classes of twists for the supergroup algebra $k \mathcal{G}$ of a finite supergroup scheme $\mathcal{G}$ over $k$ 
are parameterized by conjugacy classes of pairs $(\mathcal{H}, \mathcal{J})$, where $\mathcal{H} \subset \mathcal{G}$ is a closed supergroup subscheme and $\mathcal{J}$ is a non-degenerate twist for $k \mathcal{H}$ (just as in the case of abstract finite groups). Furthermore, using Proposition 7.5 we show in Proposition [7.6 that a twist for $\mathcal{G}$ is non-degenerate if and only if it is minimal (again, as for abstract finite groups). Finally, in Theorem 7.8 we classify finite dimensional triangular Hopf algebras with the Chevalley property over $k$.

Acknowledgments. The author is grateful to Pavel Etingof for stimulating and helpful discussions.

\section{Preliminaries}

Throughout the paper we fix an algebraically closed field $k$ of characteristic 0 or $p>2$. We refer the reader to the book [EGNO] for the general theory of tensor categories.

2.1. Affine supergroup schemes. We refer the reader to, e.g. [W], for preliminaries on affine group schemes over $k$, and to [Ma for preliminaries on affine supergroup schemes over $k$.

Let $\mathcal{G}$ be an affine supergroup scheme over $k$, with unit morphism e $: \operatorname{Spec}(k) \rightarrow \mathcal{G}$, inversion morphism i : $\mathcal{G} \rightarrow \mathcal{G}$, and multiplication morphism $\mathrm{m}: \mathcal{G} \times \mathcal{G} \rightarrow \mathcal{G}$, satisfying the usual group axioms. Recall that the coordinate algebra $\mathcal{O}(\mathcal{G})^{1}$ of $\mathcal{G}$ is a supercommutative Hopf superalgebra over $k$, and $\mathcal{G}$ is the functor from the category of supercommutative $k$-superalgebras to the category of groups defined by $R \mapsto \mathcal{G}(R):=\operatorname{Hom}_{\mathrm{SAlg}}(\mathcal{O}(\mathcal{G}), R)$ (so-called functor of points). Note that any affine supergroup scheme is the inverse limit of affine supergroup schemes of finite type.

A closed supergroup subscheme $\mathcal{H}$ of $\mathcal{G}$ is the spectrum of the Hopf quotient $\mathcal{O}(\mathcal{H}):=\mathcal{O}(\mathcal{G}) / \mathcal{I}(\mathcal{H})$ by a Hopf ideal $\mathcal{I}(\mathcal{H}) \subset \mathcal{O}(\mathcal{G})$. The ideal $\mathcal{I}(\mathcal{H})$ is referred to as the defining ideal of $\mathcal{H}$ in $\mathcal{O}(\mathcal{G})$. For example, the even part of $\mathcal{G}$ is the closed group subscheme $\mathcal{G}_{0} \subset \mathcal{G}$ with the defining ideal $\mathcal{I}\left(\mathcal{G}_{0}\right)=\left\langle\mathcal{O}(\mathcal{G})_{1}\right\rangle$, i.e., $\mathcal{G}_{0}$ is an ordinary affine group scheme with coordinate algebra $\mathcal{O}\left(\mathcal{G}_{0}\right)=\mathcal{O}(\mathcal{G}) /\left\langle\mathcal{O}(\mathcal{G})_{1}\right\rangle$. In particular, we have a surjective Hopf algebra map $\pi: \mathcal{O}(\mathcal{G}) \rightarrow \mathcal{O}\left(\mathcal{G}_{0}\right)$.

Let $\mathfrak{g}=\mathfrak{g}_{0} \oplus \mathfrak{g}_{1}$ be the Lie superalgebra of $\mathcal{G}$, i.e., $\mathfrak{g}$ is the space of left-invariant derivations of $\mathcal{O}(\mathcal{G}), \mathfrak{g}_{0}$ is the space of even derivations of $\mathcal{O}(\mathcal{G})$, and $\mathfrak{g}_{1}$ is the space of odd derivations of $\mathcal{O}(\mathcal{G})$. We have $\mathfrak{g}=\left(\mathfrak{m} / \mathfrak{m}^{2}\right)^{*}$, where $\mathfrak{m} \subset \mathcal{O}(\mathcal{G})$ is the kernel of the augmentation map, and $\mathfrak{g}_{0}=\operatorname{Lie}\left(\mathcal{G}_{0}\right)$ is the Lie algebra of $\mathcal{G}_{0}$.

\footnotetext{
${ }^{1}$ Some authors use $k[\mathcal{G}]$ instead.
} 
Recall that $\mathcal{G}_{0}$ acts on $\mathfrak{g}_{1}$ via the adjoint action. Let $a: \mathcal{G}_{0} \times \mathfrak{g}_{1}^{*} \rightarrow \mathfrak{g}_{1}^{*}$ be the coadjoint action of $\mathcal{G}_{0}$ on $\mathfrak{g}_{1}^{*}$. Then $\wedge \mathfrak{g}_{1}^{*}$ is an $\mathcal{O}\left(\mathcal{G}_{0}\right)$-comodule algebra with structure map $a^{*}: \wedge \mathfrak{g}_{1}^{*} \rightarrow \mathcal{O}\left(\mathcal{G}_{0}\right) \otimes \wedge \mathfrak{g}_{1}^{*}$.

Since $\mathcal{O}\left(\mathcal{G}_{0}\right)$ is a quotient Hopf algebra of $\mathcal{O}(\mathcal{G})$, it follows that $\mathcal{O}(\mathcal{G})$ has a canonical structure of a left $\mathcal{O}\left(\mathcal{G}_{0}\right)$-comodule algebra with structure map $(\pi \otimes \mathrm{id}) \Delta$. It is known [Ma, Theorem 4.5] that the subalgebra of $\mathcal{O}\left(\mathcal{G}_{0}\right)$-coinvariants in $\mathcal{O}(\mathcal{G})$ is isomorphic to $\wedge \mathfrak{g}_{1}^{*}$, and that we have a tensor decomposition

$$
\mathcal{O}(\mathcal{G}) \cong \wedge \mathfrak{g}_{1}^{*} \otimes \mathcal{O}\left(\mathcal{G}_{0}\right)
$$

of $\mathcal{O}\left(\mathcal{G}_{0}\right)$-supercomodule counital superalgebras. In particular, we have abelian equivalences

$$
\begin{aligned}
& \operatorname{sRep}(\mathcal{O}(\mathcal{G})) \cong \operatorname{sRep}\left(\wedge \mathfrak{g}_{1}^{*}\right) \bigotimes_{\text {svect }} \operatorname{sRep}\left(\mathcal{O}\left(\mathcal{G}_{0}\right)\right) \\
& \quad \cong \operatorname{sRep}\left(\wedge \mathfrak{g}_{1}^{*}\right) \otimes \operatorname{Rep}\left(\mathcal{O}\left(\mathcal{G}_{0}\right)\right)
\end{aligned}
$$

such that $\operatorname{Rep}\left(\mathcal{O}\left(\mathcal{G}_{0}\right)\right)$ can be identified with a full tensor subcategory of $\operatorname{sRep}(\mathcal{O}(\mathcal{G}))$ in the obvious way.

Recall that we have

$$
\operatorname{Rep}\left(\mathcal{O}\left(\mathcal{G}_{0}\right)\right)=\operatorname{Coh}_{\mathrm{f}}\left(\mathcal{G}_{0}\right)=\bigoplus_{g \in \mathcal{G}_{0}(k)} \operatorname{Coh}_{\mathrm{f}}\left(\mathcal{G}_{0}\right)_{g},
$$

where $\operatorname{Coh}_{\mathrm{f}}\left(\mathcal{G}_{0}\right)_{g}$ is the abelian subcategory of sheaves supported at $g$, with unique simple object $\delta_{g}$ and indecomposable projective object $P_{g}:=\widehat{\mathcal{O}\left(\mathcal{G}_{0}\right)_{g}}$ in the pro-completion category, where $\mathcal{O}\left(\mathcal{G}_{0}\right)_{g}$ is the completion of $\mathcal{O}\left(\mathcal{G}_{0}\right)$ at $g$ [G2, Section 3.1]. Thus by (2.2), we have

$$
\operatorname{sRep}(\mathcal{O}(\mathcal{G})) \cong \bigoplus_{g \in \mathcal{G}_{0}(k)} \operatorname{sRep}\left(\wedge \mathfrak{g}_{1}^{*}\right) \otimes \operatorname{Coh}_{\mathrm{f}}\left(\mathcal{G}_{0}\right)_{g}
$$

as abelian categories.

Recall that closed supergroup subschemes $\mathcal{H} \subset \mathcal{G}$ are in bijection with pairs $\left(\mathcal{H}_{0}, \mathfrak{h}_{1}\right)$, where $\mathcal{H}_{0} \subset \mathcal{G}_{0}$ is a closed group subscheme, $\mathfrak{h}_{1} \subset \mathfrak{g}_{1}$ is an $\mathcal{H}_{0}$-invariant subspace, and $\left[\mathfrak{h}_{1}, \mathfrak{h}_{1}\right] \subset \mathfrak{h}_{0}:=\operatorname{Lie}\left(\mathcal{H}_{0}\right)$ (see, e.g., [MS, Section 6.2]).

Let $\Psi: \mathcal{G} \times \mathcal{G} \rightarrow \mathbb{G}_{m}$ be a normalized even 2-cocycle. Equivalently, $\Psi \in \mathcal{O}(\mathcal{G}) \otimes \mathcal{O}(\mathcal{G})$ is a twist for $\mathcal{O}(\mathcal{G})$, i.e., $\Psi$ is an invertible even element satisfying the equations

$$
\begin{gathered}
(\Delta \otimes \mathrm{id})(\Psi)(\Psi \otimes 1)=(\mathrm{id} \otimes \Delta)(\Psi)(1 \otimes \Psi), \\
(\varepsilon \otimes \mathrm{id})(\Psi)=(\mathrm{id} \otimes \varepsilon)(\Psi)=1 .
\end{gathered}
$$

Finally, recall that a finite supergroup scheme $\mathcal{G}$ is an affine supergroup scheme whose function algebra $\mathcal{O}(\mathcal{G})$ is finite dimensional. In 
this case, $k \mathcal{G}:=\mathcal{O}(\mathcal{G})^{*}$ is a supercocommutative Hopf superalgebra (called the group algebra of $\mathcal{G}$ ).

2.2. Module categories over tensor categories. Let $\mathcal{C}$ be a tensor category over $k$. Let $\operatorname{Ind}(\mathcal{C})$ and $\operatorname{Pro}(\mathcal{C})$ be the categories of Ind-objects and Pro-objects of $\mathcal{C}$, respectively. It is well known that the tensor structure on $\mathcal{C}$ extends to a tensor structure on $\operatorname{Ind}(\mathcal{C})$ and $\operatorname{Pro}(\mathcal{C})$. However $\operatorname{Ind}(\mathcal{C})$ and $\operatorname{Pro}(\mathcal{C})$ are not rigid, but the rigid structure on $\mathcal{C}$ induces two duality functors $\operatorname{Pro}(\mathcal{C}) \rightarrow \operatorname{Ind}(\mathcal{C})$ ("continuous dual") and $\operatorname{Ind}(\mathcal{C}) \rightarrow \operatorname{Pro}(\mathcal{C})$ ("linear dual"), which we shall both denote by $X \mapsto X^{*}$; they are antiequivalence inverses of each other. It is also known that $\operatorname{Ind}(\mathcal{C})$ has enough injectives.

Recall that a (left) module category $\mathcal{M}$ over $\mathcal{C}$ is a locally finite abelian category equipped with a (left) action $\otimes^{\mathcal{M}}: \mathcal{C} \otimes \mathcal{M} \rightarrow \mathcal{M}$, such that the bifunctor $\otimes^{\mathcal{M}}$ is bilinear on morphisms and biexact. Recall also that $\mathcal{M}$ is exact if any additive module functor $\mathcal{M} \rightarrow \mathcal{M}_{1}$ from $\mathcal{M}$ to any other $\mathcal{C}$-module category $\mathcal{M}_{1}$ is exact, and that $\mathcal{M}$ is indecomposable if $\mathcal{M}$ is not equivalent to a direct sum of two nontrivial module subcategories. It is also known that the $\mathcal{C}$-module structure on $\mathcal{M}$ extends to a module structure on $\operatorname{Ind}(\mathcal{M})$ over $\operatorname{Ind}(\mathcal{C})$. Moreover, $\mathcal{M}$ is exact if and only if for any $M \in \mathcal{M}$ and any injective object $I \in \operatorname{Ind}(\mathcal{C})$ (resp., projective object $P \in \operatorname{Pro}(\mathcal{C})$ ), $I \otimes M$ is injective in $\operatorname{Ind}(\mathcal{M})$ (resp., $P \otimes M$ is projective in $\operatorname{Pro}(\mathcal{M})$ ) (see [EO, Propositions 3.11, 3.16], [G2, Proposition 2.4]).

Following [EO], we say that two simple objects $M_{1}, M_{2} \in \mathcal{M}$ are related if there exists an object $X \in \mathcal{C}$ such that $M_{1}$ appears as a subquotient in $X \otimes^{\mathcal{M}} M_{2}$. This defines an equivalence relation, and $\mathcal{M}$ decomposes into a direct sum $\mathcal{M}=\oplus \mathcal{M}_{i}$ of indecomposable exact module subcategories indexed by the equivalence classes (see [EO, Lemma 3.8 \& Proposition 3.9] and [G2, Proposition 2.5]).

Assume $\mathcal{M}$ is exact. Recall that an object $\delta \in \mathcal{M}$ generates $\mathcal{M}$ if for any $M \in \mathcal{M}$ there exists $X \in \mathcal{C}$ such that $\operatorname{Hom}_{\mathcal{M}}\left(X \otimes^{\mathcal{M}} \delta, M\right) \neq 0$. It is known that $\delta$ generates $\mathcal{M}$ if and only if for any $M \in \mathcal{M}$ there exists $X \in \mathcal{C}$ such that $M$ is a subquotient of $X \otimes^{\mathcal{M}} \delta$ (cf. [EO]). Thus if $\mathcal{M}$ is indecomposable and $\delta$ is simple, then $\delta \in \mathcal{M}$ generates $\mathcal{M}$.

Finally recall that for every two objects $M_{1}, M_{2} \in \mathcal{M}$, we have an object $\overline{\operatorname{Hom}}\left(M_{1}, M_{2}\right) \in \operatorname{Pro}(\mathcal{C})$ satisfying

$$
\operatorname{Hom}_{\mathcal{M}}\left(M_{2}, X \otimes^{\mathcal{M}} M_{1}\right) \cong \operatorname{Hom}_{\operatorname{Pro}(\mathcal{C})}\left(\overline{\operatorname{Hom}}\left(M_{1}, M_{2}\right), X\right), X \in \mathcal{C}
$$

(the dual internal Hom). For every $M \in \mathcal{M}$, the pro-object $\overline{\operatorname{Hom}}(M, M)$ has a canonical structure of a coalgebra. In terms of internal Hom's 
[EO], the algebra $\underline{\operatorname{Hom}}(M, M)$ in $\operatorname{Ind}(\mathcal{C})$ is isomorphic to the dual algebra $(\overline{\operatorname{Hom}}(M, M))^{*}$ under the duality functor ${ }^{*}: \operatorname{Pro}(\mathcal{C}) \rightarrow \operatorname{Ind}(\mathcal{C})$. Now if $\mathcal{M}$ is indecomposable and exact, we have a $\mathcal{C}$-module equivalence $\mathcal{M} \cong \operatorname{Comod}_{\operatorname{Pro}(\mathcal{C})}(\overline{\operatorname{Hom}}(M, M))$.

\section{The Tensor CATEgory $\operatorname{SCOH}_{\mathrm{f}}(\mathcal{G})$}

Let $\mathcal{G}$ be an affine supergroup scheme $2^{2}$ over $k$, and let

$$
\overline{\mathcal{O}(\mathcal{G})}=\mathcal{O}(\mathcal{G}) \times k\langle u\rangle
$$

be the Radford's biproduct ordinary Hopf algebra, where $u$ is a grouplike element of order 2 acting on $\mathcal{O}(\mathcal{G})$ by parity, and

$$
\bar{\Delta}(x)=\sum\left(x_{1} \otimes u^{\left|x_{2}\right|}\right) \otimes\left(x_{2} \otimes 1\right)
$$

for every homogeneous element $x \in \mathcal{O}(\mathcal{G})$, where $\Delta(x)=\sum x_{1} \otimes x_{2}$. Recall that we have an equivalence of tensor categories

$$
\operatorname{Rep}(\overline{\mathcal{O}(\mathcal{G})}) \cong \operatorname{sRep}(\mathcal{O}(\mathcal{G}))
$$

In particular, $\operatorname{Rep}\left(\mathcal{O}\left(\mathcal{G}_{0}\right)\right)$ is a tensor subcategory of $\operatorname{Rep}(\overline{\mathcal{O}(\mathcal{G})})$.

Definition 3.1. Let $\mathrm{sCoh}_{\mathrm{f}}(\mathcal{G})$ (resp., $\mathrm{sQCoh}(\mathcal{G})$ ) be the tensor category (resp., monoidal category) of finite dimensional (resp., all) representations of the Hopf algebra $\overline{\mathcal{O}(\mathcal{G})}$.

By definition, we have equivalences of tensor and monoidal categories

$$
\mathrm{sCoh}_{\mathrm{f}}(\mathcal{G}) \cong \operatorname{sRep}(\mathcal{O}(\mathcal{G})) \text { and } \operatorname{sQCoh}(\mathcal{G}) \cong \operatorname{SRep}(\mathcal{O}(\mathcal{G})) \text {, }
$$

respectively, where $\operatorname{sRep}(\mathcal{O}(\mathcal{G}))$ and $\operatorname{SRep}(\mathcal{O}(\mathcal{G}))$ are the categories of finite dimensional and all representations of the Hopf superalgebra $\mathcal{O}(\mathcal{G})$ on $k$-supervector spaces, respectively.

We have that $\operatorname{Ind}\left(\mathrm{sCoh}_{\mathrm{f}}(\mathcal{G})\right)$ is the category of locally finite quasicoherent sheaves of $\mathcal{O}(\mathcal{G})$-supermodules (i.e., representations in which every vector generates a finite dimensional subrepresentation).

Remark 3.2. By a quasi-coherent sheaf on $\mathcal{G}$ we will mean a quasicoherent sheaf of $\mathcal{O}(\mathcal{G})$-supermodules, and by a finite quasi-coherent sheaf on $\mathcal{G}$ we will mean a quasi-coherent sheaf of finite dimensional $\mathcal{O}(\mathcal{G})$-supermodules. Note that finite quasi-coherent sheaves on $\mathcal{G}$ are automatically supported on finite sets in $\mathcal{G}_{0}$. Thus, one can think of $\mathrm{sCoh}_{\mathrm{f}}(\mathcal{G})$ and $\mathrm{sQCoh}(\mathcal{G})$ as the $k$-linear abelian categories of finite quasi-coherent sheaves and quasi-coherent sheaves on $\mathcal{G}$, respectively (which explains our notation). In particular, the tensor products

\footnotetext{
${ }^{2}$ The purely even case is treated in [G2, Section 3.1].
} 
in $\mathrm{sCoh}_{\mathrm{f}}(\mathcal{G})$ and $\mathrm{sQCoh}(\mathcal{G})$ correspond to the convolution product of sheaves

$$
\mathrm{X} \otimes \mathrm{Y}:=\mathrm{m}_{*}(\mathrm{X} \otimes \mathrm{Y})
$$

(where $\mathrm{m}_{*}$ is the direct image functor of $\mathrm{m}$ ). Notice that the tensor category $\operatorname{Coh}_{\mathrm{f}}\left(\mathcal{G}_{0}\right)$ is identified with the tensor subcategory of $\operatorname{sCoh}_{\mathrm{f}}(\mathcal{G})$ consisting of sheaves on which odd elements act trivially.

We will also consider the following categories.

Definition 3.3. Let $\operatorname{Coh}_{\mathrm{f}}(\mathcal{G})$ (resp., $\mathrm{QCoh}(\mathcal{G})$ ) be the abelian category of finite dimensional (resp., all) representations of the algebra $\mathcal{O}(\mathcal{G})$.

Note that $\operatorname{Coh}_{\mathrm{f}}(\mathcal{G})$ is not a tensor category when $\mathcal{G}$ is not even, and that we have a tensor equivalence $\operatorname{sCoh}_{\mathrm{f}}\left(\mathcal{G}_{0}\right) \cong \operatorname{Coh}_{\mathrm{f}}\left(\mathcal{G}_{0}\right) \otimes$ sVect. However, we do have the following.

Lemma 3.4. The abelian category $\operatorname{Coh}_{\mathrm{f}}(\mathcal{G})$ has a natural structure of a left module category over $\mathrm{sCoh}_{\mathrm{f}}(\mathcal{G})$, given by

$$
\mathrm{sCoh}_{\mathrm{f}}(\mathcal{G}) \otimes \operatorname{Coh}_{\mathrm{f}}(\mathcal{G}) \rightarrow \operatorname{Coh}_{\mathrm{f}}(\mathcal{G}), \quad X \otimes Y \mapsto \mathrm{m}_{*}(X \otimes Y) .
$$

Moreover, the restriction functor $\mathrm{SCoh}_{\mathrm{f}}(\mathcal{G}) \rightarrow \mathrm{Coh}_{\mathrm{f}}(\mathcal{G})$, induced by the algebra inclusion $\mathcal{O}(\mathcal{G}) \subset \overline{\mathcal{O}(\mathcal{G})}$, has a canonical structure of a surjective $\mathrm{sCoh}_{\mathrm{f}}(\mathcal{G})$-module functor.

Proof. Since by $(3.1), \mathcal{O}(\mathcal{G}) \subset \overline{\mathcal{O}(\mathcal{G})}$ is a left coideal subalgebra, the claim follows from (3.2).

For every $g \in \mathcal{G}_{0}(k)$, let $\operatorname{sCoh}_{\mathrm{f}}(\mathcal{G})_{g}:=\operatorname{sRep}\left(\wedge \mathfrak{g}_{1}^{*}\right) \otimes \operatorname{Coh}_{\mathrm{f}}\left(\mathcal{G}_{0}\right)_{g}$. By (2.2), we have an abelian equivalence

$$
\mathrm{sCoh}_{\mathrm{f}}(\mathcal{G}) \cong \bigoplus_{g \in \mathcal{G}_{0}(k)} \operatorname{sCoh}_{\mathrm{f}}(\mathcal{G})_{g} .
$$

We will need the following result.

Lemma 3.5. Every tensor subcategory of $\mathrm{sCoh}_{\mathrm{f}}(\mathcal{G})$ is either of the form $\mathrm{SCoh}_{\mathrm{f}}(\mathcal{H})$ or $\operatorname{Coh}_{\mathrm{f}}(\mathcal{H})$ for some closed supergroup subscheme $\mathcal{H} \subset \mathcal{G}$ or closed subgroup scheme $\mathcal{H} \subset \mathcal{G}_{0}$, respectively.

Proof. It is known that every tensor subcategory of $\operatorname{Rep}(\overline{\mathcal{O}(\mathcal{G})})$ corresponds to a Hopf quotient of $\overline{\mathcal{O}(\mathcal{G})}$. Now if $u$ is mapped to 1 in the quotient, then we get the second case (as all odd elements must act by zero). Otherwise, we get the first case.

Remark 3.6. The class of tensor categories $\operatorname{sCoh}_{\mathrm{f}}(\mathcal{G})$ can be extended to a larger class of tensor categories $\operatorname{sCoh}_{\mathrm{f}}(\mathcal{G}, \Omega)$ in exactly the same way as in the even case [G2, Section 5]. Namely, let $\mathcal{G}$ be an affine 
supergroup scheme over $k$, and let $\Omega \in Z^{3}\left(\mathcal{G}, \mathbb{G}_{m}\right)$ be a normalized even 3-cocycle. Equivalently, $\Omega$ is a Drinfeld associator for $\mathcal{O}(\mathcal{G})$, i.e., $\Omega \in \mathcal{O}(\mathcal{G})^{\otimes 3}$ is an invertible even element satisfying the equations

$$
(\mathrm{id} \otimes \mathrm{id} \otimes \Delta)(\Omega)(\Delta \otimes \mathrm{id} \otimes \mathrm{id})(\Omega)=(1 \otimes \Omega)(\mathrm{id} \otimes \Delta \otimes \mathrm{id})(\Omega)(\Omega \otimes 1)
$$

and

$$
(\varepsilon \otimes \mathrm{id} \otimes \mathrm{id})(\Omega)=(\mathrm{id} \otimes \varepsilon \otimes \mathrm{id})(\Omega)=(\mathrm{id} \otimes \mathrm{id} \otimes \varepsilon)(\Omega)=1 .
$$

Then $\operatorname{sCoh}_{\mathrm{f}}(\mathcal{G}, \Omega)$ is the abelian category $\operatorname{sCoh}_{\mathrm{f}}(\mathcal{G})$ equipped with the tensor product given by convolution and associativity constraint given by the action of $\Omega$ (viewed as an invertible element in $\mathcal{O}(\mathcal{G})^{\otimes 3}$ ).

\section{EqUIVARIANT QUASI-COHERENT SHEAVES}

Let $\mathcal{G}$ be an affine supergroup scheme 3 over $k$, let $\mathcal{H} \subset \mathcal{G}$ be a closed supergroup subscheme (see 2.1), and let $\iota=\iota_{\mathcal{H}}: \mathcal{H} \hookrightarrow \mathcal{G}$ be the inclusion morphism. Let $\mu: \mathcal{G} \times \mathcal{H} \rightarrow \mathcal{G}$ be the free action of $\mathcal{H}$ on $\mathcal{G}$ by right translations (in other words, the free actions of $\mathcal{H}(R)$ on $\mathcal{G}(R)$ by right translations that are functorial in $R, R$ a supercommutative $k$-superalgebra). Set

$$
\eta:=\mu(\mathrm{id} \times \mathrm{m})=\mu(\mu \times \mathrm{id}): \mathcal{G} \times \mathcal{H} \times \mathcal{H} \rightarrow \mathcal{G},
$$

and let

$$
\mathrm{p}_{1}: \mathcal{G} \times \mathcal{H} \rightarrow \mathcal{G}, \mathrm{p}_{1}: \mathcal{G} \times \mathcal{H} \times \mathcal{H} \rightarrow \mathcal{G}, \mathrm{p}_{12}: \mathcal{G} \times \mathcal{H} \times \mathcal{H} \rightarrow \mathcal{G} \times \mathcal{H}
$$

be the obvious projections. We clearly have $\mathrm{p}_{1} \circ \mathrm{p}_{12}=\mathrm{p}_{1}$ as morphisms $\mathcal{G} \times \mathcal{H} \times \mathcal{H} \rightarrow \mathcal{G}$.

Now let $\Psi: \mathcal{H} \times \mathcal{H} \rightarrow \mathbb{G}_{m}$ be a normalized even 2-cocycle, i.e., $\Psi \in \mathcal{O}(\mathcal{H})^{\otimes 2}$ is a twist for $\mathcal{O}(\mathcal{H})$ (see 2.1), and let $\mathcal{O}(\mathcal{H})_{\Psi}$ be the ("twisted") supercoalgebra with underlying supervector space $\mathcal{O}(\mathcal{H})$ and comultiplication $\Delta_{\Psi}$ given by $\Delta_{\Psi}(f):=\Delta(f) \Psi$, where $\Delta$ is the standard comultiplication of $\mathcal{O}(\mathcal{H})$. Note that $\Psi$ defines an automorphism of any quasi-coherent sheaf on $\mathcal{H} \times \mathcal{H}$ by multiplication.

Definition 4.1. Let $\Psi: \mathcal{H} \times \mathcal{H} \rightarrow \mathbb{G}_{m}$ be a normalized even 2-cocycle on a closed supergroup subscheme $\mathcal{H} \subset \mathcal{G}$.

(1) $\operatorname{An}(\mathcal{H}, \Psi)$-equivariant quasi-coherent sheaf on $\mathcal{G}$ is a pair $(S, \lambda)$, where $S \in \operatorname{sQCoh}(\mathcal{G})$ and $\lambda: \mathrm{p}_{1}^{*}(S) \stackrel{\cong}{\rightarrow} \mu^{*}(S)$ is an isomorphism

\footnotetext{
${ }^{3}$ The purely even case is treated in [G2, Section 3.2].
} 
of sheaves on $\mathcal{G} \times \mathcal{H}$, such that the diagram of morphisms of sheaves on $\mathcal{G} \times \mathcal{H} \times \mathcal{H}$

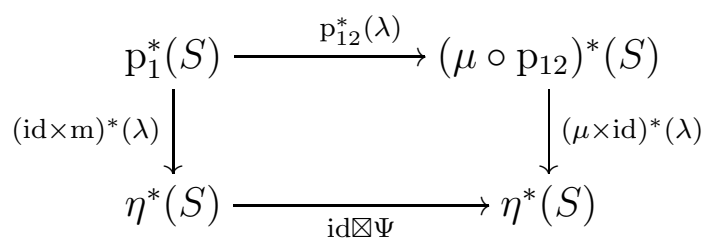

is commutative.

(2) Let $\left(S, \lambda_{S}\right)$ and $\left(T, \lambda_{T}\right)$ be two $(\mathcal{H}, \Psi)$-equivariant quasi-coherent sheaves on $\mathcal{G}$. A morphism $\phi: S \rightarrow T$ in $\operatorname{sQCoh}(\mathcal{G})$ is said to be $(\mathcal{H}, \Psi)$-equivariant if the diagram of morphisms of sheaves on $\mathcal{G} \times \mathcal{H}$

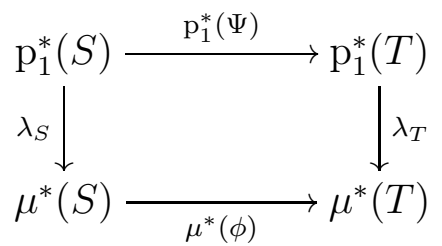

is commutative.

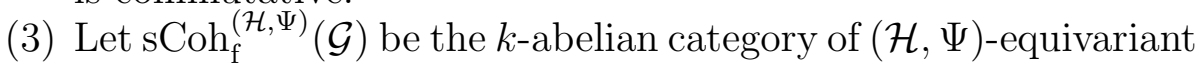
coherent sheaves on $\mathcal{G}$ with finite support in $\mathcal{G}_{0} / \mathcal{H}_{0}$ (i.e., sheaves supported on finitely many $\mathcal{H}_{0}$-cosets), with $(\mathcal{H}, \Psi)$-equivariant morphisms.

Replacing $\mathrm{sQCoh}(\mathcal{G})$ with $\mathrm{QCoh}(\mathcal{G})$ everywhere in Definition 4.1, we define the notion of an $(\mathcal{H}, \Psi)$-equivariant $\mathcal{O}(\mathcal{G})$-module, and the $k$ abelian category $\operatorname{Coh}_{\mathrm{f}}^{(\mathcal{H}, \Psi)}(\mathcal{G})$ of finitely generated $(\mathcal{H}, \Psi)$-equivariant $\mathcal{O}(\mathcal{G})$-modules with finite support in $\mathcal{G}_{0} / \mathcal{H}_{0}$.

Example 4.2. We have

$$
\operatorname{SCoh}_{\mathrm{f}}^{(\{1\}, 1)}(\mathcal{G})=\operatorname{sCoh}_{\mathrm{f}}(\mathcal{G}) \text { and } \operatorname{Coh}_{\mathrm{f}}^{(\{1\}, 1)}(\mathcal{G})=\operatorname{Coh}_{\mathrm{f}}(\mathcal{G}) \text {. }
$$

Remark 4.3. Let $\left(\mathcal{H}^{\prime}, \Psi^{\prime}\right)$ be another pair consisting of a closed supergroup subscheme $\mathcal{H}^{\prime} \subset \mathcal{G}$ and an even normalized 2-cocycle $\Psi^{\prime}$ on $\mathcal{H}^{\prime}$. By considering the free right action of $\mathcal{H}^{\prime} \times \mathcal{H}$ on $\mathcal{G}$ given by $g(a, b):=$ $a^{-1} g b$, we can similarly define $\left(\left(\mathcal{H}^{\prime}, \Psi^{\prime}\right),(\mathcal{H}, \Psi)\right)$-biequivariant quasicoherent sheaves on $\mathcal{G},\left(\left(\mathcal{H}^{\prime}, \Psi^{\prime}\right),(\mathcal{H}, \Psi)\right)$-equivariant $\mathcal{O}(\mathcal{G})$-modules, and the $k$-abelian categories $\operatorname{sCoh}_{\mathrm{f}}^{\left(\left(\mathcal{H}^{\prime}, \Psi^{\prime}\right),(\mathcal{H}, \Psi)\right)}(\mathcal{G}), \operatorname{Coh}_{\mathrm{f}}^{\left(\left(\mathcal{H}^{\prime}, \Psi^{\prime}\right),(\mathcal{H}, \Psi)\right)}(\mathcal{G})$.

Remark 4.4. Retain the notation from Remark 3.6. Let $\mathcal{H} \subset \mathcal{G}$ be a closed supergroup subscheme, and let $\Psi \in C^{2}\left(\mathcal{H}, \mathbb{G}_{m}\right)$ be a normalized even 2-cochain such that $d \Psi=\Omega_{\mid \mathcal{H}}$. Then similarly to $\operatorname{sCoh}_{\mathrm{f}}^{(\mathcal{H}, \Psi)}(\mathcal{G})$ 
(the case $\Omega=1$ ), with the obvious adjustments, we can define the category $\operatorname{sCoh}_{\mathrm{f}}^{(\mathcal{H}, \Psi)}(\mathcal{G}, \Omega)$ of $(\mathcal{H}, \Psi)$-equivariant coherent sheaves on $(\mathcal{G}, \Omega)$ with finite support in $\mathcal{G}_{0} / \mathcal{H}_{0}$, and the category $\operatorname{Coh}_{\mathrm{f}}^{(\mathcal{H}, \Psi)}(\mathcal{G}, \Omega)$ of finitely generated $(\mathcal{H}, \Psi)$-equivariant $\mathcal{O}(\mathcal{G}, \Omega)$-modules, where $\mathcal{O}(\mathcal{G}, \Omega)$ is the obviously defined quasi-Hopf algebra.

Consider now the supercoalgebra $\mathcal{O}(\mathcal{H})_{\Psi}$ in $\operatorname{sCoh}(\mathcal{G})$, and let $\widehat{\mathcal{O H})_{\Psi}}$ be its profinite completion with respect to the superalgebra structure of $\mathcal{O}(\mathcal{H})$ (see [G2, Example 2.4]). Then $\widehat{\mathcal{O}(\mathcal{H})_{\Psi}}$ is a supercoalgebra object in both $\operatorname{Pro}(\mathrm{sCoh}(\mathcal{G}))$ and $\operatorname{Pro}\left(\operatorname{sCoh}_{\mathrm{f}}(\mathcal{G})\right)$.

Lemma 4.5. We have abelian equivalences

$$
\operatorname{sCoh}_{\mathrm{f}}^{(\mathcal{H}, \Psi)}(\mathcal{G}) \cong \operatorname{Comod}_{\operatorname{Pro}\left(\operatorname{sCoh}_{\mathrm{f}}(\mathcal{G})\right)}\left(\widehat{\mathcal{O}(\mathcal{H})_{\Psi}}\right)
$$

and

$$
\operatorname{Coh}_{\mathrm{f}}^{(\mathcal{H}, \Psi)}(\mathcal{G}) \cong \operatorname{Comod}_{\operatorname{Pro}\left(\operatorname{Coh}_{\mathrm{f}}(\mathcal{G})\right)}(\widehat{\mathcal{O ( H )}}) .
$$

Proof. We prove the first equivalence, the proof of the second one being similar.

For every $S \in \operatorname{Pro}\left(\operatorname{sCoh}_{\mathrm{f}}(\mathcal{G})\right)$, we have a natural isomorphism

$$
\operatorname{Hom}_{\mathcal{G} \times \mathcal{H}}\left(\mu^{*}(S), \mathrm{p}_{1}^{*}(S)\right) \cong \operatorname{Hom}_{\mathcal{G}}\left(S, \mu_{*} \mathrm{p}_{1}^{*}(S)\right)
$$

("adjunction"). Since $\mu_{*} \mathrm{p}_{1}^{*}(S) \cong S \otimes \widehat{\mathcal{O}(\mathcal{H})}$, we can assign to any isomorphism $\lambda: \mu^{*}(S) \rightarrow \mathrm{p}_{1}^{*}(S)$ a morphism $\rho_{\lambda}: S \rightarrow S \otimes \widehat{\mathcal{O}(\mathcal{H})}$. It is now straightforward to verify that $\rho_{\lambda}: S \rightarrow S \otimes \widehat{\mathcal{O}(\mathcal{H})_{\Psi}}$ is a comodule map if and only if $\left(S, \lambda^{-1}\right)$ is an $(\mathcal{H}, \Psi)$-equivariant coherent sheaf on $\mathcal{G}$ with finite support in $\mathcal{G}_{0} / \mathcal{H}_{0}$.

The next proposition will be very useful in the sequel.

Proposition 4.6. Let $\mathcal{H} \subset \mathcal{G}$ be a closed supergroup subscheme, and let $\Psi$ be an even normalized 2-cocycle on $\mathcal{H}$. Then the following hold:

(1) The structure sheaf $\mathcal{O}(\mathcal{H})$ of $\mathcal{H}$ admits a canonical structure of an $(\mathcal{H}, \Psi)$-equivariant coherent sheaf on $\mathcal{H}$, making it a simple object of $\mathrm{sCoh}_{\mathrm{f}}^{(\mathcal{H}, \Psi)}(\mathcal{H}) \cong$ sVect, and the regular $\mathcal{O}(\mathcal{H})$-module admits a canonical structure of an $(\mathcal{H}, \Psi)$-equivariant $\mathcal{O}(\mathcal{H})$ module, making it the simple object of $\operatorname{Coh}_{\mathrm{f}}^{(\mathcal{H}, \Psi)}(\mathcal{H}) \cong$ Vect.

(2) The sheaf $\iota_{*} \mathcal{O}(\mathcal{H})^{4}$ is a simple object in $\mathrm{sCoh}_{\mathrm{f}}^{(\mathcal{H}, \Psi)}(\mathcal{G})$, and the $\mathcal{O}(\mathcal{G})$-module $\iota_{*} \mathcal{O}(\mathcal{H}) \in \operatorname{Coh}(\mathcal{G})$ 同 is a simple object in $\operatorname{Coh}_{\mathrm{f}}^{(\mathcal{H}, \Psi)}(\mathcal{G})$.

\footnotetext{
${ }^{4}$ The superrepresentation of $\mathcal{O}(\mathcal{G})$ on $\mathcal{O}(\mathcal{H})$ coming from $\iota$.

${ }^{5}$ The representation of $\mathcal{O}(\mathcal{G})$ on $\mathcal{O}(\mathcal{H})$ coming from $\iota$.
} 
(3) For every $X \in \operatorname{sCoh}_{\mathrm{f}}(\mathcal{G})$, we have

$$
\mathrm{m}_{*}(X \otimes M) \in \operatorname{sCoh}_{\mathrm{f}}^{(\mathcal{H}, \Psi)}(\mathcal{G}), M \in \operatorname{sCoh}_{\mathrm{f}}^{(\mathcal{H}, \Psi)}(\mathcal{G}),
$$

and

$$
\mathrm{m}_{*}(X \otimes M) \in \operatorname{Coh}_{\mathrm{f}}^{(\mathcal{H}, \Psi)}(\mathcal{G}), M \in \operatorname{Coh}_{\mathrm{f}}^{(\mathcal{H}, \Psi)}(\mathcal{G}) .
$$

Proof. We will prove the proposition for sheaves, the proof for modules being similar.

(1) Consider the isomorphism $\varphi:=\left(\mathrm{m}, \mathrm{p}_{2}\right): \mathcal{H} \times \mathcal{H} \cong \mathcal{H} \times \mathcal{H}$. Since $\mathrm{p}_{1} \circ \varphi=\mathrm{m}$, it follows that $\left(\mathrm{p}_{1} \circ \varphi\right)^{*} \mathcal{O}(\mathcal{H})=\mathrm{m}^{*} \mathcal{O}(\mathcal{H})$. Now, multiplication by $\Psi$ defines an isomorphism

$$
\mathrm{m}^{*} \mathcal{O}(\mathcal{H})=\left(\mathrm{p}_{1} \circ \varphi\right)^{*} \mathcal{O}(\mathcal{H})=\left(\varphi^{*} \circ \mathrm{p}_{1}^{*}\right) \mathcal{O}(\mathcal{H}) \stackrel{\Psi}{\rightarrow}\left(\varphi^{*} \circ \mathrm{p}_{1}^{*}\right) \mathcal{O}(\mathcal{H})
$$

and since we have $\mathrm{p}_{1}^{*} \mathcal{O}(\mathcal{H})=\mathcal{O}(\mathcal{H}) \otimes \mathcal{O}(\mathcal{H})$, we get an isomorphism

$$
\lambda: \mathrm{p}_{1}^{*} \mathcal{O}(\mathcal{H})=\mathcal{O}(\mathcal{H}) \otimes \mathcal{O}(\mathcal{H}) \stackrel{\varphi^{*}}{\rightarrow} \varphi^{*}(\mathcal{O}(\mathcal{H}) \otimes \mathcal{O}(\mathcal{H})) \stackrel{\Psi^{-1}}{\longrightarrow} \mathrm{m}^{*} \mathcal{O}(\mathcal{H}) .
$$

The fact that $(\mathcal{O}(\mathcal{H}), \lambda)$ is an $(\mathcal{H}, \Psi)$-equivariant coherent sheaf on $\mathcal{H}$ can be checked now in a straightforward manner using the tensor decomposition (2.1). Clearly, $(\mathcal{O}(\mathcal{H}), \lambda)$ is a simple object in $\operatorname{sCoh}_{\mathrm{f}}^{(\mathcal{H}, \Psi)}(\mathcal{H})$.

Let $\delta:=(\mathcal{O}(\mathcal{H}), \lambda)$, and consider the simple object $\delta^{-}:=k^{0 \mid 1} \otimes \delta$ (via id $\otimes \Delta$ ). It is clear that $\delta \nsubseteq \delta^{-}$in $\operatorname{sCoh}_{\mathrm{f}}^{(\mathcal{H}, \Psi)}(\mathcal{H})$.

Now let $M$ be any object in $\operatorname{sCoh}_{\mathrm{f}}^{(\mathcal{H}, \Psi)}(\mathcal{H})$, and let $X:=M^{\operatorname{coO}(\mathcal{H})}$. We claim that

$$
M \cong M^{\operatorname{co} \mathcal{O}(\mathcal{H})} \otimes_{k} \delta:=\bar{X}_{0} \otimes_{k} \delta \oplus \bar{X}_{1} \otimes_{k} \delta^{-}
$$

in $\mathrm{sCoh}_{\mathrm{f}}^{(\mathcal{H}, \Psi)}(\mathcal{H})$, where $\bar{X}$ denotes the underlying vector space of $X$. Indeed, let $\alpha: M^{\operatorname{coO}(\mathcal{H})} \otimes_{k} \mathcal{O}(\mathcal{H}) \rightarrow M$ be the action map, and let

$$
\beta: M \rightarrow M^{\operatorname{co} \mathcal{O}(\mathcal{H})} \otimes_{k} \mathcal{O}(\mathcal{H}), m \mapsto \sum S^{-1}\left(m_{1}\right) \cdot m_{0} \otimes m_{2} .
$$

Then it is straightforward to check that $\alpha$ and $\beta$ are inverse to each other. Hence, $\operatorname{sCoh}_{\mathrm{f}}^{(\mathcal{H}, \Psi)}(\mathcal{H})$ is semisimple of rank 2, as claimed.

(2) Since $\iota$ is affine, the commutative diagrams
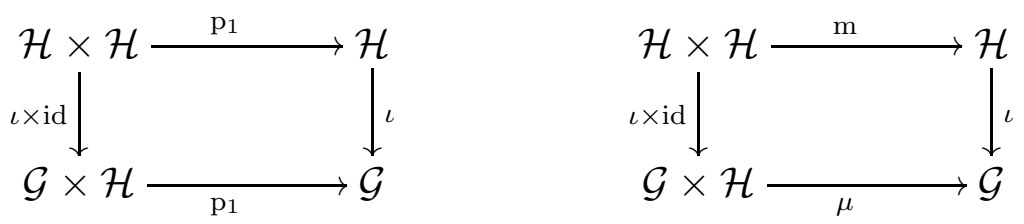

yield isomorphisms

$$
\mathrm{p}_{1}^{*} \iota_{*} \mathcal{O}(\mathcal{H}) \stackrel{\cong}{\rightarrow}(\iota \times \mathrm{id})_{*} \mathrm{p}_{1}^{*} \mathcal{O}(\mathcal{H})
$$


and

$$
(\iota \times \mathrm{id})_{*} \mathrm{~m}^{*} \mathcal{O}(\mathcal{H}) \stackrel{\cong}{\rightarrow} \mu^{*} \iota_{*} \mathcal{O}(\mathcal{H})
$$

("base change").

Let $\lambda: \mathrm{p}_{1}^{*} \mathcal{O}(\mathcal{H}) \stackrel{\cong}{\rightarrow} \mathrm{m}^{*} \mathcal{O}(\mathcal{H})$ be the isomorphism constructed in Part (1). Since $\iota$ is $\mathcal{H}$-equivariant, we get an isomorphism

$$
(\iota \times \mathrm{id})_{*} \mathrm{p}_{1}^{*} \mathcal{O}(\mathcal{H}) \stackrel{(\iota \times \mathrm{id})_{*}(\lambda)}{\longrightarrow}(\iota \times \mathrm{id})_{*} \mathrm{~m}^{*} \mathcal{O}(\mathcal{H}) .
$$

It is now straightforward to check, using the tensor decomposition (2.1), that the composition of isomorphisms (4.1), (4.2) and (4.3)

$$
\mathrm{p}_{1}^{*} \iota_{*} \mathcal{O}(\mathcal{H}) \stackrel{\cong}{\rightarrow} \mu^{*} \iota_{*} \mathcal{O}(\mathcal{H})
$$

endows $\iota_{*} \mathcal{O}(\mathcal{H})$ with a structure of an $(\mathcal{H}, \Psi)$-equivariant coherent sheaf on $\mathcal{G}$. Clearly, $\iota_{*} \mathcal{O}(\mathcal{H})$ is simple.

(3) Consider the right action id $\times \mu: \mathcal{G} \times \mathcal{G} \times \mathcal{H} \rightarrow \mathcal{G} \times \mathcal{G}$ of $\mathcal{H}$ on $\mathcal{G} \times \mathcal{G}$. If $M \in \operatorname{sCoh}_{\mathrm{f}}^{(\mathcal{H}, \Psi)}(\mathcal{G})$, it is clear that $X \otimes M \in \operatorname{sCoh}_{\mathrm{f}}(\mathcal{G} \times \mathcal{G})$ is an $(\mathcal{H}, \Psi)$-equivariant coherent sheaf on $\mathcal{G} \times \mathcal{G}$ (here we identify $\mathcal{H}$ with the supergroup subscheme $\{1\} \times \mathcal{H} \subset \mathcal{G} \times \mathcal{G})$. But since $\mathrm{m}: \mathcal{G} \times \mathcal{G} \rightarrow \mathcal{G}$ is $\mathcal{H}$-equivariant, $\mathrm{m}_{*}$ carries $(\mathcal{H}, \Psi)$-equivariant coherent sheaves on $\mathcal{G} \times \mathcal{G}$ to $(\mathcal{H}, \Psi)$-equivariant coherent sheaves on $\mathcal{G}$.

\section{Exact module CATEgories OVER $\operatorname{sCoh}_{\mathrm{f}}(\mathcal{G})$}

In this section we extend [G2, Section 3.3] to the super case.

Let $\mathcal{G}, \mathcal{H}, \iota$ and $\Psi$ be as in 4 , Set

$$
\mathcal{M}=\mathcal{M}(\mathcal{H}, \Psi):=\operatorname{sCoh}_{\mathrm{f}}^{(\mathcal{H}, \Psi)}(\mathcal{G}), \quad \mathcal{M}^{\circ}=\mathcal{M}^{\circ}(\mathcal{H}, \Psi):=\operatorname{Coh}_{\mathrm{f}}^{(\mathcal{H}, \Psi)}(\mathcal{G}),
$$

and let

$$
\begin{aligned}
& \mathcal{V}=\mathcal{V}(\mathcal{H}, \Psi):=\operatorname{Comod}_{\operatorname{Pro}\left(\mathrm{sCoh}_{\mathrm{f}}(\mathcal{G})\right)}\left(\widehat{\mathcal{O}(\mathcal{H})_{\Psi}}\right), \\
& \mathcal{V}^{\circ}=\mathcal{V}^{\circ}(\mathcal{H}, \Psi):=\operatorname{Comod}_{\operatorname{Pro}\left(\operatorname{Coh}_{\mathrm{f}}(\mathcal{G})\right)}\left(\widehat{\mathcal{O}(\mathcal{H})_{\Psi}}\right)
\end{aligned}
$$

be the abelian categories of right comodules over $\widehat{\mathcal{O}(\mathcal{H})_{\Psi}}$ in $\operatorname{Pro}\left(\operatorname{sCoh}_{\mathrm{f}}(\mathcal{G})\right)$ and $\operatorname{Pro}\left(\operatorname{Coh}_{\mathrm{f}}(\mathcal{G})\right)$, respectively.

Let

$\delta=\delta_{\mathcal{H}}:=\iota_{*} \mathcal{O}(\mathcal{H}) \in \operatorname{sCoh}_{\mathrm{f}}^{(\mathcal{H}, \Psi)}(\mathcal{G}), \quad \delta^{\circ}=\delta_{\mathcal{H}}^{\circ}:=\iota_{*} \mathcal{O}(\mathcal{H}) \in \operatorname{Coh}_{\mathrm{f}}^{(\mathcal{H}, \Psi)}(\mathcal{G})$.

Proposition 5.1. The following hold:

(1) The bifunctors

$$
\begin{aligned}
& \otimes^{\mathcal{M}}: \operatorname{sCoh}_{\mathrm{f}}(\mathcal{G}) \otimes \mathcal{M} \rightarrow \mathcal{M}, X \otimes M \mapsto \mathrm{m}_{*}(X \otimes M) \\
& \text { and } \\
& \otimes^{\mathcal{M}^{\circ}}: \operatorname{sCoh}_{\mathrm{f}}(\mathcal{G}) \otimes \mathcal{M}^{\circ} \rightarrow \mathcal{M}^{\circ}, X \otimes M \mapsto \mathrm{m}_{*}(X \otimes M)
\end{aligned}
$$


define on $\mathcal{M}$ and $\mathcal{M}^{\circ}$ structures of indecomposable $\operatorname{sCoh}_{\mathrm{f}}(\mathcal{G})$ module categories.

(2) The bifunctors

$$
\otimes^{\mathcal{V}}: \mathrm{sCoh}_{\mathrm{f}}(\mathcal{G}) \otimes \mathcal{V} \rightarrow \mathcal{V}, X \otimes V \mapsto \mathrm{m}_{*}(X \otimes V)
$$

and

$$
\otimes \mathcal{V}^{\circ}: \operatorname{sCoh}_{\mathrm{f}}(\mathcal{G}) \otimes \mathcal{V}^{\circ} \rightarrow \mathcal{V}^{\circ}, X \otimes V \mapsto \mathrm{m}_{*}(X \otimes V)
$$

define on $\mathcal{V}$ and $\mathcal{V}^{\circ}$ structures of $\mathrm{sCoh}_{\mathrm{f}}(\mathcal{G})$-module categories.

(3) We have equivalences $\mathcal{M} \cong \mathcal{V}$ and $\mathcal{M}^{\circ} \cong \mathcal{V}^{\circ}$ of module categories over $\operatorname{sCoh}_{\mathrm{f}}(\mathcal{G})$. In particular, $\overline{\operatorname{Hom}}(\delta, \delta) \cong \widehat{\mathcal{O}(\mathcal{H})_{\Psi}}$ as supercoalgebras in $\operatorname{Pro}\left(\operatorname{sCoh}_{\mathrm{f}}(\mathcal{G})\right)$, and $\overline{\operatorname{Hom}}\left(\delta^{\circ}, \delta^{\circ}\right) \cong \widehat{\mathcal{O}(\mathcal{H})_{\Psi}}$ as coalgebras in $\operatorname{Pro}\left(\operatorname{Coh}_{\mathrm{f}}(\mathcal{G})\right)$.

Proof. We prove it for $\mathcal{M}$ and $\mathcal{V}$, the proof for $\mathcal{M}^{\circ}$ and $\mathcal{V}^{\circ}$ being similar.

(1) Since $\mathrm{m}(\mathrm{m} \times \mathrm{id})=\mathrm{m}(\mathrm{id} \times \mathrm{m})$ and $\Psi$ is an even 2-cocycle, it follows from Lemma 4.6 that $\otimes^{\mathcal{M}}$ defines on $\mathcal{M}$ a structure of $\operatorname{sCoh}_{\mathrm{f}}(\mathcal{G})$ module category. Clearly, $\mathrm{sCoh}_{\mathrm{f}}(\mathcal{H}) \subset \mathrm{sCoh}_{\mathrm{f}}(\mathcal{G})$ consists of those objects $X$ for which $X \otimes^{\mathcal{M}} \delta$ is a sum of multiples of $\delta$ and $k^{0 \mid 1} \otimes \delta$, and any object $M \in \mathcal{M}$ is of the form $X \otimes^{\mathcal{M}} \delta$ for some $X \in \operatorname{sCoh}_{\mathrm{f}}(\mathcal{G})$. In particular, the simple object $\delta$ (see Proposition 4.6) generates $\mathcal{M}$, so $\mathcal{M}$ is indecomposable.

(2) By definition, an object in $\mathcal{V}$ is a pair $\left(V, \rho_{V}\right)$ consisting of an object $V \in \operatorname{Pro}\left(\operatorname{sCoh}_{\mathrm{f}}(\mathcal{G})\right)$ and a morphism $\rho_{V}: V \rightarrow V \otimes \widehat{\mathcal{O}(\mathcal{H})_{\Psi}}$ in $\operatorname{Pro}\left(\mathrm{sCoh}_{\mathrm{f}}(\mathcal{G})\right)$ satisfying the comodule axioms. It is clear that for

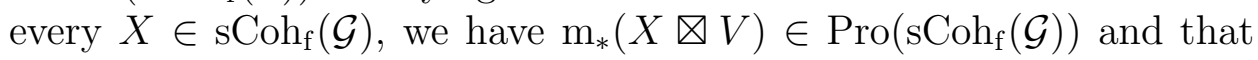
$\rho_{\mathrm{m}_{*}(X \otimes V)}:=\operatorname{id}_{X} \otimes \rho_{V}$ is a morphism in $\operatorname{Pro}\left(\operatorname{sCoh}_{\mathrm{f}}(\mathcal{G})\right)$ defining on $\mathrm{m}_{*}(X \otimes V)$ a structure of a right comodule over $\widehat{\mathcal{O}(\mathcal{H})_{\Psi}}$.

(3) Follows from Lemma 4.5.

Example 5.2. Let $\mathcal{G}$ be an affine supergroup scheme over $k$.

(1) $\mathcal{M}(\{1\}, 1)=\operatorname{sCoh}_{\mathrm{f}}(\mathcal{G})$ (the regular module).

(2) $\mathcal{M}^{\circ}(\{1\}, 1)=\operatorname{Coh}_{\mathrm{f}}(\mathcal{G})$.

(3) $\mathcal{M}(\mathcal{G}, 1)=\operatorname{sVect}\left(\right.$ the standard superfiber functor on $\mathrm{sCoh}_{\mathrm{f}}(\mathcal{G})$ ).

(4) $\mathcal{M}^{\circ}(\mathcal{G}, 1)=\operatorname{Vect}\left(\right.$ the standard fiber functor on $\operatorname{sCoh}_{\mathrm{f}}(\mathcal{G})$ ).

Proposition 5.3. The indecomposable module categories $\mathcal{M}(\mathcal{H}, \Psi)$ and $\mathcal{M}^{\circ}(\mathcal{H}, \Psi)$ over $\mathrm{sCoh}_{\mathrm{f}}(\mathcal{G})$ are exact.

Proof. We prove it for $\mathcal{M}(\mathcal{H}, \Psi)$, the proof for $\mathcal{M}^{\circ}(\mathcal{H}, \Psi)$ being similar.

Set $\mathcal{M}:=\mathcal{M}(\mathcal{H}, \Psi)$. It suffices to show that for every projective $P \in$ $\operatorname{Pro}\left(\operatorname{sCoh}_{\mathrm{f}}(\mathcal{G})\right)$ and $X \in \mathcal{M}, P \otimes^{\mathcal{M}} X$ is projective (see 2.2). Clearly, it suffices to show it for $X:=\delta=\delta_{(\mathcal{H}, \Psi)}$. Moreover, since any projective 
in $\operatorname{Pro}\left(\operatorname{sCoh}_{\mathrm{f}}(\mathcal{G})\right)$ is a completed direct sum of $P_{g, \pm}$ (see 3$)$, it suffices to check that $P_{g} \otimes^{\mathcal{M}} \delta$ is projective. Furthermore, since $P_{g}=\delta_{g} \otimes P_{1}$, and $\delta_{g} \otimes^{\mathcal{M}}$ ? is an autoequivalence of $\mathcal{M}$ as an abelian category (since $\delta_{g}$ is invertible), it suffices to do so for $g=1$. Finally, this is done just by computing this product explicitly using the definition, which yields that $P_{1} \otimes^{\mathcal{M}} \delta=\widehat{\mathcal{O}(\mathcal{H})_{1}} \otimes_{k} P(\delta)$, where $P(\delta)$ is the projective cover of $\delta$ (i.e., the unique indecomposable projective in the block of $\operatorname{Pro}(\mathcal{M})$ containing $\delta$; as a sheaf on $\mathcal{G}$, it is the function algebra on the formal neighborhood of $\mathcal{H}$ ), and hence projective as desired.

We say that two pairs $(\mathcal{H}, \Psi)$ and $\left(\mathcal{H}^{\prime}, \Psi^{\prime}\right)$ are conjugate if there exists $g \in \mathcal{G}_{0}(k)$ such that $g \mathcal{H} g^{-1}=\mathcal{H}^{\prime}$ and $\Psi^{g}=\Psi^{\prime}$ in $H^{2}\left(\mathcal{H}^{\prime}, \mathbb{G}_{m}\right)$.

Lemma 5.4. If $(\mathcal{H}, \Psi)$ and $\left(\mathcal{H}^{\prime}, \Psi^{\prime}\right)$ are conjugate then

$$
\mathcal{M}(\mathcal{H}, \Psi) \cong \mathcal{M}\left(\mathcal{H}^{\prime}, \Psi^{\prime}\right) \text { and } \mathcal{M}^{\circ}(\mathcal{H}, \Psi) \cong \mathcal{M}^{\circ}\left(\mathcal{H}^{\prime}, \Psi^{\prime}\right)
$$

as module categories over $\operatorname{sCoh}_{\mathrm{f}}(\mathcal{G})$.

Proof. Follows from Proposition 5.1 since for every $g \in \mathcal{G}_{0}(k)$, we have $\widehat{\mathcal{O}(\mathcal{H})_{\Psi}} \cong \mathcal{O}\left(\widehat{g \mathcal{H} g^{-1}}\right)_{\Psi^{g}}$ as supercoalgebras in $\operatorname{Pro}\left(\operatorname{sCoh}_{\mathrm{f}}(\mathcal{G})\right)$.

We are now ready to state and prove the main result of this section.

Theorem 5.5. Let $\mathcal{G}$ be an affine supergroup scheme over $k$. There is a 1 : 2 correspondence between conjugacy classes of pairs $(\mathcal{H}, \Psi)$ and equivalence classes of indecomposable exact module categories over $\operatorname{sCoh}_{\mathrm{f}}(\mathcal{G})$, assigning $(\mathcal{H}, \Psi)$ to $\mathcal{M}(\mathcal{H}, \Psi)$ and $\mathcal{M}^{\circ}(\mathcal{H}, \Psi)$.

Proof. By Proposition 5.3 and Lemma 5.4, it remains to show that any indecomposable exact module category $\mathcal{M}$ over $\operatorname{sCoh}_{\mathrm{f}}(\mathcal{G})$ is of the form $\mathcal{M}(\mathcal{H}, \Psi)$ or $\mathcal{M}^{\circ}(\mathcal{H}, \Psi)$ for a unique pair $(\mathcal{H}, \Psi)$ (up to conjugation). To this end, pick a simple object $\delta$ in $\mathcal{M}$, and let $\delta^{-}:=k^{0 \mid 1} \otimes^{\mathcal{M}} \delta$. There are two cases: either $\delta \nsubseteq \delta^{-}$or $\delta \cong \delta^{-}$.

Assume that $\delta \not \delta^{-}$. Consider the full subcategory

$\mathcal{C}:=\left\{X \in \operatorname{sCoh}_{\mathrm{f}}(\mathcal{G}) \mid X \otimes \otimes^{\mathcal{M}} \delta=\operatorname{dim}_{k}\left(X_{0}\right) \delta \oplus \operatorname{dim}_{k}\left(X_{1}\right) \delta^{-}\right\} \subset \operatorname{sCoh}_{\mathrm{f}}(\mathcal{G})$.

For every $X, Y \in \mathcal{C}$, we have

$$
\begin{aligned}
&(X \otimes Y) \otimes^{\mathcal{M}} \delta \cong X \otimes^{\mathcal{M}}\left(Y \otimes^{\mathcal{M}} \delta\right) \\
& \cong X \otimes^{\mathcal{M}}\left(\operatorname{dim}_{k}\left(Y_{0}\right) \delta \oplus \operatorname{dim}_{k}\left(Y_{1}\right) \delta^{-}\right) \\
& \cong \operatorname{dim}_{k}\left(Y_{0}\right) X \otimes^{\mathcal{M}} \delta \oplus \operatorname{dim}_{k}\left(Y_{1}\right) \Pi(X) \otimes^{\mathcal{M}} \delta \\
& \cong \operatorname{dim}_{k}\left(Y_{0}\right)\left(\operatorname{dim}_{k}\left(X_{0}\right) \delta \oplus \operatorname{dim}_{k}\left(X_{1}\right) \delta^{-}\right) \oplus \\
& \operatorname{dim}_{k}\left(Y_{1}\right)\left(\operatorname{dim}_{k}\left(X_{1}\right) \delta \oplus \operatorname{dim}_{k}\left(X_{0}\right) \delta^{-}\right) \\
&= \operatorname{dim}_{k}\left(Y_{0} \otimes X_{0} \oplus Y_{1} \otimes X_{1}\right) \delta \oplus \operatorname{dim}_{k}\left(Y_{0} \otimes X_{1} \oplus Y_{1} \otimes X_{0}\right) \delta^{-} .
\end{aligned}
$$


Thus, $\mathcal{C} \subset \mathrm{sCoh}_{\mathrm{f}}(\mathcal{G})$ is a tensor subcategory. Moreover, the functor

$$
F: \mathcal{C} \rightarrow \operatorname{Vect}, F(X)=\operatorname{Hom}_{\mathcal{M}}\left(\delta \oplus \delta^{-}, X \otimes^{\mathcal{M}} \delta\right),
$$

together with the tensor structure $F(\cdot) \otimes F(\cdot) \stackrel{\cong}{\rightarrow} F(\cdot \otimes \cdot)$ coming from the associativity constraint, is a fiber functor on $\mathcal{C}$.

Now by Lemma 3.5, either $\mathcal{C}=\mathrm{sCoh}_{\mathrm{f}}(\mathcal{H})$ for some closed supergroup subscheme $\mathcal{H} \subset \mathcal{G}$, or $\mathcal{C}=\operatorname{Coh}_{\mathrm{f}}(\mathcal{H})$ for some closed group subscheme $\mathcal{H} \subset \mathcal{G}_{0}$. In any case, we see that for every $X \in \mathcal{C}, F(X)=\bar{X}$ is the underlying vector space of $X$. Thus we have a functorial isomorphism $\bar{X} \otimes \bar{Y} \cong \overline{X \otimes Y}$, which is nothing but an invertible even element $\Psi$ of $\mathcal{O}(\mathcal{H})^{\otimes 2}$, taking values in $\mathbb{G}_{m}(k)$. Clearly, $\Psi$ is a twist for $\mathcal{O}(\mathcal{H})$.

Thus, we have obtained that if $\mathcal{C}=\operatorname{sCoh}_{\mathrm{f}}(\mathcal{H})$ then the $\mathcal{C}$-submodule category $\langle\delta\rangle \subset \mathcal{M}$ is equivalent to $\operatorname{sCoh}_{\mathrm{f}}^{(\mathcal{H}, \Psi)}(\mathcal{H})$, and if $\mathcal{C}=\operatorname{Coh}_{\mathrm{f}}(\mathcal{H})$ then the $\mathcal{C}$-submodule category $\langle\delta\rangle \subset \mathcal{M}$ is equivalent to $\operatorname{Coh}_{\mathrm{f}}^{(\mathcal{H}, \Psi)}(\mathcal{H})$.

For $X \in \mathrm{sCoh}_{\mathrm{f}}(\mathcal{G})$, let $X_{\mathcal{H}} \in \mathcal{C}$ be the maximal subsheaf of $X$ which is scheme-theoretically supported on $\mathcal{H}$ (i.e., $\overline{X_{\mathcal{H}}}$ consists of all vectors in $\bar{X}$ which are annihilated by the defining ideal of $\mathcal{H}$ in $\mathcal{O}(\mathcal{G})$ ). Now, on the one hand, since for any $g \in \mathcal{G}_{0}(k), \delta_{g} \otimes^{\mathcal{M}} \delta$ and $\delta_{g} \otimes^{\mathcal{M}} \delta^{-}$are simple, and one of them is isomorphic to $\delta$ and the other one to $\delta^{-}$if and only if $g \in \mathcal{H}_{0}(k)$, it is clear that

$$
\begin{aligned}
& \operatorname{Hom}_{\operatorname{Pro}\left(\mathrm{sCoh}_{\mathrm{f}}(\mathcal{G})\right)}\left(\overline{\operatorname{Hom}}\left(\delta \oplus \delta^{-}, \delta \oplus \delta^{-}\right), X\right) \\
& =\operatorname{Hom}_{\mathcal{M}}\left(\delta \oplus \delta^{-}, X \otimes^{\mathcal{M}}\left(\delta \oplus \delta^{-}\right)\right)=\overline{X_{\mathcal{H}}}
\end{aligned}
$$

(since it holds for any simple $X$ ). On the other hand, it is clear that

$$
\operatorname{Hom}_{\operatorname{Pro}\left(\mathrm{sCoh}_{\mathrm{f}}(\mathcal{G})\right)}\left(\widehat{\mathcal{O}(\mathcal{H})_{\Psi}}, X\right)=\overline{X_{\mathcal{H}}} \text {. }
$$

Thus by Yoneda's lemma, the two supercoalgebras $\overline{\operatorname{Hom}}\left(\delta \oplus \delta^{-}, \delta \oplus \delta^{-}\right)$ and $\widehat{\mathcal{O}(\mathcal{H})_{\Psi}}$ are isomorphic in $\operatorname{Pro}\left(\mathrm{sCoh}_{\mathrm{f}}(\mathcal{G})\right)$. This implies that $\mathcal{M}$ is equivalent to $\operatorname{Comod}_{\operatorname{Pro}\left(\mathrm{sCoh}_{\mathrm{f}}(\mathcal{G})\right)}\left(\widehat{\mathcal{O}(\mathcal{H})_{\Psi}}\right)$ as a module category over $\mathrm{sCoh}_{\mathrm{f}}(\mathcal{G})$ (as $\mathcal{M}$ is indecomposable, exact, and generated by $\delta \oplus \delta^{-}$), hence to $\mathcal{M}(\mathcal{H}, \Psi)$ by Proposition 5.1, as desired.

Furthermore, the conjugacy class of $(\mathcal{H}, \Psi)$ is uniquely determined by $\mathcal{M}$ since replacing $\delta$ with $\delta_{g} \otimes^{\mathcal{M}} \delta, g \in \mathcal{G}_{0}(k)$, corresponds to replacing $\mathcal{H}$ with $g \mathcal{H} g^{-1}$ and $\Psi$ with $\Psi^{g}$.

Finally, if $\delta \cong \delta^{-}$then the proof that $\mathcal{M} \cong \mathcal{M}^{\circ}(\mathcal{H}, \Psi)$ as module categories over $\mathrm{sCoh}_{\mathrm{f}}(\mathcal{G})$ for a uniquely determined conjugacy class of pairs, is similar.

Example 5.6. Since for an affine group scheme $\mathcal{G}$ over $k$, we have $\mathrm{sCoh}_{\mathrm{f}}(\mathcal{G})=\operatorname{Coh}_{\mathrm{f}}(\mathcal{G}) \otimes \mathrm{sVect}$, we see that Theorem 5.5 reduces to G2, Theorem 3.9] in the even case. 
Example 5.7. Let $V$ be a $n$-dimensional odd $k$-vector space, $n \geq 0$. By Theorem 5.5, equivalence classes of indecomposable exact module categories over $\operatorname{sCoh}_{\mathrm{f}}(V)$ are in $2: 1$ correspondence with equivalence classes of pairs $(W, B)$, where $W \subset V$ is a super subspace and $B \in S^{2} W^{*}$. For example, if $n=0$ then there are two non-equivalent indecomposable exact module categories over $\mathrm{sCoh}_{\mathrm{f}}(V)=$ sVect: Vect and sVect. Also, if $n=1$ then there are exactly three non-equivalent pairs of the form $(W, B):(0,0),(V, 0)$ and $(V, B)$, where $B(v, v)=1$ $(v$ a fixed basis for $V$ ). Thus, there are six non-equivalent indecomposable exact module categories over $\mathrm{sCoh}_{\mathrm{f}}(V)$ (in agreement with [EO, Theorem 4.5]). More precisely, we have $\mathcal{M}(0,0) \cong \operatorname{sCoh}_{\mathrm{f}}(V)$ and $\mathcal{M}^{\circ}(0,0) \cong \operatorname{Coh}_{\mathrm{f}}(V), \mathcal{M}(V, 0), \mathcal{M}(V, B)$, which are semisimple of rank 2, and $\mathcal{M}^{\circ}(V, 0), \mathcal{M}^{\circ}(V, B)$, which are semisimple of rank 1 .

Remark 5.8. Retain the notation from Remark 4.4. Similarly, the categories $\operatorname{sCoh}_{\mathrm{f}}^{(\mathcal{H}, \Psi)}(\mathcal{G}, \Omega)$ and $\operatorname{Coh}_{\mathrm{f}}^{(\mathcal{H}, \Psi)}(\mathcal{G}, \Omega)$ admit a structure of an indecomposable exact module category over $\operatorname{sCoh}_{\mathrm{f}}(\mathcal{G}, \Omega)$ given by convolution of sheaves, and furthermore, there is a $1: 2$ correspondence between (appropriately defined) conjugacy classes of pairs $(\mathcal{H}, \Psi)$ and equivalence classes of indecomposable exact module categories over $\operatorname{sCoh}_{\mathrm{f}}(\mathcal{G}, \Omega)$, assigning $(\mathcal{H}, \Psi)$ to $\operatorname{sCoh}_{\mathrm{f}}^{(\mathcal{H}, \Psi)}(\mathcal{G}, \Omega)$ and $\operatorname{Coh}_{\mathrm{f}}^{(\mathcal{H}, \Psi)}(\mathcal{G}, \Omega)$.

\section{Exact module CATEgories OVER $\operatorname{sRep}(\mathcal{G})$}

In this section we extend [G2, Section 4] to the super case.

Let $\mathcal{C}$ be a tensor category. Given two exact module categories $\mathcal{M}, \mathcal{N}$ over $\mathcal{C}$, let $\operatorname{Fun}_{\mathcal{C}}(\mathcal{M}, \mathcal{N})$ denote the abelian category of $\mathcal{C}$-functors from $\mathcal{M}$ to $\mathcal{N}$. The dual category of $\mathcal{C}$ with respect to $\mathcal{M}$ is the category $\mathcal{C}_{\mathcal{M}}^{*}:=\operatorname{End}_{\mathcal{C}}(\mathcal{M})$ of $\mathcal{C}$-endofunctors of $\mathcal{M}$. If $\mathcal{M}$ is indecomposable, $\mathcal{C}_{\mathcal{M}}^{*}$ is a tensor category, and $\mathcal{M}$ is an indecomposable exact module category over $\mathcal{C}_{\mathcal{M}}^{*}$. Also, $\operatorname{Fun}_{\mathcal{C}}(\mathcal{M}, \mathcal{N})$ is an exact module category over $\mathcal{C}_{\mathcal{M}}^{*}$ via the composition of functors.

6.1. Module categories. Retain the notation from 4 and 5. Set

$$
\mathcal{M}((\mathcal{G}, 1),(\mathcal{H}, \Psi)):=\operatorname{sCoh}_{\mathrm{f}}^{((\mathcal{G}, 1),(\mathcal{H}, \Psi))}(\mathcal{G})
$$

and

$$
\mathcal{M}^{\circ}((\mathcal{G}, 1),(\mathcal{H}, \Psi)):=\operatorname{Coh}_{\mathrm{f}}^{((\mathcal{G}, 1),(\mathcal{H}, \Psi))}(\mathcal{G}) .
$$

Recall that the 2-cocycle $\Psi$ determines a central extension $\mathcal{H}_{\Psi}$ of $\mathcal{H}$ by $\mathbb{G}_{m}$. By an $(\mathcal{H}, \Psi)$-superrepresentation of $\mathcal{H}$ we will mean a rational representation of the affine supergroup scheme $\mathcal{H}_{\Psi}$ on a $k$ supervector space on which $\mathbb{G}_{m}$ acts with weight 1 (i.e., via the identity 
character). Let us denote the category of finite dimensional $(\mathcal{H}, \Psi)$ superrepresentations of $\mathcal{H}_{\Psi}$ by $\mathcal{N}(\mathcal{H}, \Psi)$. Clearly, we have an equivalence of abelian categories

$$
\mathcal{N}(\mathcal{H}, \Psi) \cong \operatorname{sComod}\left(\mathcal{O}(\mathcal{H})_{\Psi}\right) .
$$

Similarly, let $\mathcal{N}^{\circ}(\mathcal{H}, \Psi)$ be the category of finite dimensional $(\mathcal{H}, \Psi)$ representations of $\mathcal{H}_{\Psi}$. We have an equivalence of abelian categories

$$
\mathcal{N}^{\circ}(\mathcal{H}, \Psi) \cong \operatorname{Comod}\left(\mathcal{O}(\mathcal{H})_{\Psi}\right) \text {. }
$$

Lemma 6.1. The following hold:

(1) We have abelian equivalences

$$
\operatorname{Fun}_{\mathrm{S} \operatorname{Coh}_{\mathrm{f}}(\mathcal{G})}\left(\mathcal{M}^{\circ}(\mathcal{G}, 1), \mathcal{M}(\mathcal{H}, \Psi)\right) \cong \mathcal{M}^{\circ}((\mathcal{G}, 1),(\mathcal{H}, \Psi))
$$

and

$$
\operatorname{Fun}_{\mathrm{sCoh}_{\mathrm{f}}(\mathcal{G})}\left(\mathcal{M}^{\circ}(\mathcal{G}, 1), \mathcal{M}^{\circ}(\mathcal{H}, \Psi)\right) \cong \mathcal{M}((\mathcal{G}, 1),(\mathcal{H}, \Psi)) .
$$

In particular, we have a tensor equivalence

$$
\operatorname{sCoh}_{\mathrm{f}}(\mathcal{G})_{\mathcal{M}^{\circ}(\mathcal{G}, 1)}^{*} \cong \operatorname{sRep}(\mathcal{G}) \text {. }
$$

(2) We have $\operatorname{sRep}(\mathcal{G})$-module equivalences

$$
\operatorname{Fun}_{\mathrm{S} \operatorname{Coh}_{\mathrm{f}}(\mathcal{G})}\left(\mathcal{M}^{\circ}(\mathcal{G}, 1), \mathcal{M}(\mathcal{H}, \Psi)\right) \cong \mathcal{N}^{\circ}(\mathcal{H}, \Psi)
$$

and

$$
\operatorname{Fun}_{\mathrm{SCoh}}(\mathcal{G})\left(\mathcal{M}^{\circ}(\mathcal{G}, 1), \mathcal{M}^{\circ}(\mathcal{H}, \Psi)\right) \cong \mathcal{N}(\mathcal{H}, \Psi) .
$$

Proof. We prove the theorem for functors to $\mathcal{M}(\mathcal{H}, \Psi)$, the proof for functors to $\mathcal{M}^{\circ}(\mathcal{H}, \Psi)$ being similar.

(1) Since $\mathcal{M}^{\circ}(\mathcal{G}, 1)=$ Vect, a functor $\mathcal{M}^{\circ}(\mathcal{G}, 1) \rightarrow \mathcal{M}(\mathcal{H}, \Psi)$ is just an $(\mathcal{H}, \Psi)$-equivariant sheaf $X$ on $\mathcal{G}$. The fact that the functor is a $\mathrm{sCoh}_{\mathrm{f}}(\mathcal{G})$-module functor means that we have functorial isomorphisms $\mu_{S}: \bar{S} \otimes X \stackrel{\cong}{\rightarrow} S \otimes X$ in $\mathcal{M}(\mathcal{H}, \Psi), S \in \operatorname{sCoh}_{\mathrm{f}}(\mathcal{G})$. Thus, $\mu$ gives $X$ a commuting $\mathcal{G}$-equivariant structure for the left action of $\mathcal{G}$ on itself, i.e., $X$ is a $((\mathcal{G}, 1),(\mathcal{H}, \Psi))$-biequivariant sheaf on $\mathcal{G}$. In particular, for $S=k^{0 \mid 1}$, we have an isomorphism $\mu_{k^{0 \mid 1}}: X_{0} \cong X_{1}$, hence $X$ corresponds to $((\mathcal{G}, 1),(\mathcal{H}, \Psi))$-biequivariant $\mathcal{O}(\mathcal{G})$-module, as desired.

Conversely, it is clear that any $((\mathcal{G}, 1),(\mathcal{H}, \Psi))$-biequivariant $\mathcal{O}(\mathcal{G})$ module $X_{0}$ defines a $\operatorname{sCoh}_{\mathrm{f}}(\mathcal{G})$-module functor $\mathcal{M}^{\circ}(\mathcal{G}, 1) \rightarrow \mathcal{M}(\mathcal{H}, \Psi)$ determined by $k \mapsto X$ with $X_{0}=X_{1}$.

Finally, the category of $(\mathcal{G}, \mathcal{G})$-biequivariant sheaves on $\mathcal{G}$ is equivalent to the category $\operatorname{sep}(\mathcal{G})$ as a tensor category, and the second claim follows.

(2) If $X$ is a $((\mathcal{G}, 1),(\mathcal{H}, \Psi))$-biequivariant $\mathcal{O}(\mathcal{G})$-module, then the inverse image sheaf $\mathrm{e}^{*}(X)$ on $\operatorname{Spec}(k)$ ("the stalk at 1 ") acquires a 
structure of an $(\mathcal{H}, \Psi)$-representation via the action of the element $\left(h, h^{-1}\right)$ in $\mathcal{G} \times \mathcal{H}$, i.e., it is an object in $\mathcal{N}^{\circ}(\mathcal{H}, \Psi)$. We have thus defined a functor

$$
\mathcal{M}^{\circ}((\mathcal{G}, 1),(\mathcal{H}, \Psi)) \rightarrow \mathcal{N}^{\circ}(\mathcal{H}, \Psi), X \mapsto \mathrm{e}^{*}(X) .
$$

Conversely, an $(\mathcal{H}, \Psi)$-representation $V$ can be spread out over $\mathcal{G}$ and made into a $(\mathcal{G},(\mathcal{H}, \Psi))$-biequivariant $\mathcal{O}(\mathcal{G})$-module. In other words, we have the functor

$$
\mathcal{N}^{\circ}(\mathcal{H}, \Psi) \rightarrow \mathcal{M}^{\circ}((\mathcal{G}, 1),(\mathcal{H}, \Psi)), V \mapsto \mathcal{O}(\mathcal{G}) \otimes_{k} V .
$$

Finally, it is straightforward to verify that the two functors constructed above are inverse to each other.

Similarly, we have the following result.

\section{Lemma 6.2. The following hold:}

(1) We have abelian equivalences

$$
\operatorname{Fun}_{\mathrm{s} \mathrm{Coh}_{\mathrm{f}}(\mathcal{G})}(\mathcal{M}(\mathcal{G}, 1), \mathcal{M}(\mathcal{H}, \Psi)) \cong \mathcal{M}((\mathcal{G}, 1),(\mathcal{H}, \Psi))
$$

and

$\operatorname{Fun}_{\mathrm{s} \operatorname{Coh}_{\mathrm{f}}(\mathcal{G})}\left(\mathcal{M}(\mathcal{G}, 1), \mathcal{M}^{\circ}(\mathcal{H}, \Psi)\right) \cong \mathcal{M}^{\circ}((\mathcal{G}, 1),(\mathcal{H}, \Psi))$.

In particular, we have a tensor equivalence

$$
\mathrm{sCoh}_{\mathrm{f}}(\mathcal{G})_{\mathcal{M}(\mathcal{G}, 1)}^{*} \cong \operatorname{sRep}(\mathcal{G}) .
$$

(2) We have $\operatorname{sRep}(\mathcal{G})$-module equivalences

$$
\begin{aligned}
& \operatorname{Fun}_{\mathrm{s} \operatorname{Coh}_{\mathrm{f}}(\mathcal{G})}(\mathcal{M}(\mathcal{G}, 1), \mathcal{M}(\mathcal{H}, \Psi)) \cong \mathcal{N}(\mathcal{H}, \Psi) \\
& \operatorname{Fun}_{\mathrm{SCoh}_{\mathrm{f}}(\mathcal{G})}\left(\mathcal{M}(\mathcal{G}, 1), \mathcal{M}^{\circ}(\mathcal{H}, \Psi)\right) \cong \mathcal{N}^{\circ}(\mathcal{H}, \Psi) .
\end{aligned}
$$

Example 6.3. We have the following:

(1) $\mathcal{N}(\{1\}, 1)=$ sVect is the usual superfiber functor on $\operatorname{sRep}(\mathcal{G})$.

(2) $\mathcal{N}^{\circ}(\{1\}, 1)=$ Vect is the usual fiber functor on $\operatorname{sRep}(\mathcal{G})$.

Lemma 6.4. The following hold:

(1) We have a tensor equivalence

$$
\operatorname{sRep}(\mathcal{G})_{\mathcal{N}^{\circ}(\{1\}, 1)}^{*} \cong \operatorname{sCoh}_{\mathrm{f}}(\mathcal{G}) .
$$

(2) We have $\operatorname{sCoh}_{\mathrm{f}}(\mathcal{G})$-module equivalences

$$
\operatorname{Fun}_{\operatorname{sRep}(\mathcal{G})}\left(\mathcal{N}^{\circ}(\{1\}, 1), \mathcal{N}(\mathcal{H}, \Psi)\right) \cong \mathcal{M}^{\circ}(\mathcal{H}, \Psi)
$$

and

$$
\operatorname{Fun}_{\operatorname{sRep}(\mathcal{G})}\left(\mathcal{N}^{\circ}(\{1\}, 1), \mathcal{N}^{\circ}(\mathcal{H}, \Psi)\right) \cong \mathcal{M}(\mathcal{H}, \Psi) .
$$


Proof. The proof is similar to the proof of Lemma 6.1.

Lemma 6.4 prompts the following definition.

Definition 6.5. An indecomposable exact module category $\mathcal{N}$ over $\operatorname{sRep}(\mathcal{G})$ is called geometrical if $\operatorname{Fun}_{\operatorname{sRep}(\mathcal{G})}\left(\mathcal{N}^{\circ}(\{1\}, 1), \mathcal{N}\right) \neq 0$.

It is clear that geometrical module categories over $\operatorname{sRep}(\mathcal{G})$ form a full 2-subcategory $\operatorname{Mod}_{\text {geom }}(\operatorname{sRep}(\mathcal{G}))$ of the 2-category $\operatorname{Mod}(\operatorname{sRep}(\mathcal{G}))$.

We can now deduce from Lemmas 6.1, 6.4 the main result of this section, which says that geometrical module categories over $\operatorname{sRep}(\mathcal{G})$ are precisely those exact module categories which come from exact module categories over $\mathrm{sCoh}_{\mathrm{f}}(\mathcal{G})$. More precisely, we have the following generalization of [G2, Theorem 4.5].

Recall that $(\mathcal{H}, \Psi)$ and $\left(\mathcal{H}^{\prime}, \Psi^{\prime}\right)$ are conjugate if there exists $g \in \mathcal{G}_{0}(k)$ such that $g \mathcal{H} g^{-1}=\mathcal{H}^{\prime}$ and $\Psi^{g}=\Psi^{\prime}$ in $H^{2}\left(\mathcal{H}^{\prime}, \mathbb{G}_{m}\right)$.

Theorem 6.6. Let $\mathcal{G}$ be an affine supergroup scheme over $k$. Then the 2-functors

$\operatorname{Mod}\left(\operatorname{sCoh}_{\mathrm{f}}(\mathcal{G})\right) \rightarrow \operatorname{Mod}_{\text {geom }}(\operatorname{sRep}(\mathcal{G})), \mathcal{M} \mapsto \operatorname{Fun}_{\mathrm{sCoh}_{\mathrm{f}}(\mathcal{G})}\left(\mathcal{M}^{\circ}(\mathcal{G}, 1), \mathcal{M}\right)$, and

$\operatorname{Mod}_{\text {geom }}(\operatorname{sRep}(\mathcal{G})) \rightarrow \operatorname{Mod}\left(\operatorname{sCoh}_{\mathrm{f}}(\mathcal{G})\right), \mathcal{N} \mapsto \operatorname{Fun}_{\mathrm{sRep}(\mathcal{G})}\left(\mathcal{N}^{\circ}(\{1\}, 1), \mathcal{N}\right)$, are inverse to each other. In particular, there is a $1: 2$ correspondence between conjugacy classes of pairs $(\mathcal{H}, \Psi)$ and equivalence classes of indecomposable geometrical module categories over $\operatorname{sRep}(\mathcal{G})$, assigning $(\mathcal{H}, \Psi)$ to $\mathcal{N}(\mathcal{H}, \Psi)$ and $\mathcal{N}^{\circ}(\mathcal{H}, \Psi)$.

Remark 6.7. If $\mathcal{G}$ is not finite, $\operatorname{sRep}(\mathcal{G})$ may very well have nongeometrical module categories (see [G2, Remark 4.6]).

Remark 6.8. Retain the notation from Remark 5.8. Similarly to the even case [G2], we can define supergroup scheme-theoretical categories $\mathcal{C}(\mathcal{G}, \mathcal{H}, \Omega, \Psi)$ and $\mathcal{C}^{\circ}(\mathcal{G}, \mathcal{H}, \Omega, \Psi)$ as the dual categories of $\operatorname{sCoh}_{\mathrm{f}}(\mathcal{G}, \Omega)$ with respect to $\operatorname{SCoh}_{\mathrm{f}}^{(\mathcal{H}, \Psi)}(\mathcal{G}, \Omega)$ and $\left.\operatorname{Coh}_{\mathrm{f}}^{(\mathcal{H}, \Psi)}(\mathcal{G}, \Omega)\right)$, respectively. We then have that $\mathcal{C}(\mathcal{G}, \mathcal{H}, \Omega, \Psi)$ is equivalent to the tensor category of $((\mathcal{H}, \Psi),(\mathcal{H}, \Psi))$-biequivariant coherent sheaves on $(\mathcal{G}, \Omega)$, supported on finitely many left $\mathcal{H}_{0}$-cosets (equivalently, right $\mathcal{H}_{0}$-cosets), with tensor product given by convolution of sheaves. For example, the center $\mathcal{Z}\left(\mathrm{sCoh}_{\mathrm{f}}(\mathcal{G})\right)$ of $\mathrm{sCoh}_{\mathrm{f}}(\mathcal{G})$ is supergroup scheme-theoretical since

$$
\mathcal{Z}\left(\operatorname{sCoh}_{\mathrm{f}}(\mathcal{G})\right) \cong\left(\operatorname{sCoh}_{\mathrm{f}}(\mathcal{G}) \otimes \operatorname{sCoh}_{\mathrm{f}}(\mathcal{G})^{\mathrm{op}}\right)_{\mathrm{SCoh}_{\mathrm{f}}(\mathcal{G})}^{*}
$$

SO

$$
\mathcal{Z}\left(\mathrm{sCoh}_{\mathrm{f}}(\mathcal{G})\right) \cong \mathcal{C}(\mathcal{G} \times \mathcal{G}, \mathcal{G}, 1,1)
$$


as tensor categories, where $\mathcal{G}$ is viewed as a closed supergroup subscheme of $\mathcal{G} \times \mathcal{G}$ via the diagonal morphism $\Delta: \mathcal{G} \rightarrow \mathcal{G} \times \mathcal{G}$.

Moreover, we can define indecomposable geometrical module categories over $\mathcal{C}:=\mathcal{C}(\mathcal{G}, \mathcal{H}, \Omega, \Psi)$, and obtain that the 2-functors

$\operatorname{Mod}\left(\operatorname{sCoh}_{\mathrm{f}}(\mathcal{G}, \Omega)\right) \rightarrow \operatorname{Mod}_{\text {geom }}(\mathcal{C}), \mathcal{M} \mapsto \operatorname{Fun}_{\mathrm{s} C o h_{\mathrm{f}}(\mathcal{G}, \Omega)}(\mathcal{M}(\mathcal{H}, \Psi), \mathcal{M})$, and

$$
\operatorname{Mod}_{\text {geom }}(\mathcal{C}) \rightarrow \operatorname{Mod}\left(\operatorname{sCoh}_{\mathrm{f}}(\mathcal{G}, \Omega)\right), \mathcal{N} \mapsto \operatorname{Fun}_{\mathcal{C}}(\mathcal{M}(\mathcal{H}, \Psi), \mathcal{N}),
$$

are 2-equivalences which are inverse to each other. In particular, the equivalence classes of geometrical module categories over $\mathcal{C}$ are in $2: 1$ correspondence with the conjugacy classes of pairs $\left(\mathcal{H}^{\prime}, \Psi^{\prime}\right)$ such that $\mathcal{H}^{\prime} \subset \mathcal{G}$ is a closed supergroup subscheme and $\Psi^{\prime} \in C^{2}\left(\mathcal{H}^{\prime}, \mathbb{G}_{m}\right)$ satisfies $d \Psi^{\prime}=\Omega_{\mid \mathcal{H}^{\prime}}$. (The analogs for $\mathcal{C}^{\circ}(\mathcal{G}, \mathcal{H}, \Omega, \Psi)$ are obvious.)

6.2. Semisimple module categories of rank 1. Recall that the set of equivalence classes of semisimple module categories over $\operatorname{sep}(\mathcal{G})$ of rank 1 is in bijection with the set of equivalence classes of tensor structures on the forgetful functor $\operatorname{sRep}(\mathcal{G}) \rightarrow$ Vect. Therefore, Theorem 6.6 implies that the conjugacy class of any pair $(\mathcal{H}, \Psi)$ for which the category $\operatorname{sComod}\left(\mathcal{O}(\mathcal{H})_{\Psi}\right)$ or $\operatorname{Comod}\left(\mathcal{O}(\mathcal{H})_{\Psi}\right)$ is semisimple of rank 1 gives rise to an equivalence class of a tensor structure on the forgetful functor $\operatorname{sRep}(\mathcal{G}) \rightarrow$ Vect. Clearly, for such pair $(\mathcal{H}, \Psi), \mathcal{H}$ must be a finite supergroup subscheme of $\mathcal{G}$ (as a simple coalgebra must be finite dimensional). This observation suggests the following definition.

Definition 6.9. Let $\mathcal{H}$ be a finite supergroup scheme over $k$. We call an even 2-cocycle $\Psi: \mathcal{H} \times \mathcal{H} \rightarrow \mathbb{G}_{m}$ (equivalently, a twist $\Psi$ for $\left.\mathcal{O}(\mathcal{H})=(k \mathcal{H})^{*}\right)$ non-degenerate if the category $\operatorname{sComod}\left(\mathcal{O}(\mathcal{H})_{\Psi}\right)$ or $\operatorname{Comod}\left(\mathcal{O}(\mathcal{H})_{\Psi}\right)$ is equivalent to Vect.

We thus have the following corollary.

Corollary 6.10. The conjugacy class of a pair $(\mathcal{H}, \Psi)$, where $\mathcal{H} \subset \mathcal{G}$ is a finite closed supergroup subscheme and $\Psi: \mathcal{H} \times \mathcal{H} \rightarrow \mathbb{G}_{m}$ is a non-degenerate even 2-cocycle, gives rise to an equivalence class of an even Hopf 2-cocycle for $\mathcal{O}(\mathcal{G})$.

Remark 6.11. Finite supergroup schemes having a non-degenerate even 2-cocycle may be called supergroup schemes of central type in analogy with the even case [G2, Remark 4.9].

6.3. Exact module categories over finite supergroup schemes. Thanks to [EO, Theorem 3.31], Theorem 6.6 can be strengthened in the finite case to give a canonical bijection between exact module categories over $\operatorname{sCoh}_{\mathrm{f}}(\mathcal{G})=\operatorname{sCoh}(\mathcal{G})$ and $\operatorname{sRep}(\mathcal{G})$ (i.e., for finite supergroup 
schemes, every exact module category over $\operatorname{sRep}(\mathcal{G})$ is geometrical). Namely, we have the following result.

Theorem 6.12. Let $\mathcal{G}$ be a finite supergroup scheme over $k$. The 2functors

$$
\operatorname{Mod}(\operatorname{sCoh}(\mathcal{G})) \rightarrow \operatorname{Mod}(\operatorname{sRep}(\mathcal{G})), \mathcal{M} \mapsto \operatorname{Fun}_{\mathrm{s} \operatorname{Coh}(\mathcal{G})}\left(\mathcal{M}^{\circ}(\mathcal{G}, 1), \mathcal{M}\right),
$$

and

$$
\operatorname{Mod}(\operatorname{sRep}(\mathcal{G})) \rightarrow \operatorname{Mod}(\operatorname{sCoh}(\mathcal{G})), \mathcal{N} \mapsto \operatorname{Fun}_{\mathrm{sRep}(\mathcal{G})}\left(\mathcal{N}^{\circ}(\{1\}, 1), \mathcal{N}\right),
$$

are inverse to each other. In particular, the equivalence classes of indecomposable exact module categories over $\operatorname{sep}(\mathcal{G})=\operatorname{sRep}(k \mathcal{G})$ are $2: 1$ parameterized by the conjugacy classes of pairs $(\mathcal{H}, \Psi)$, where $\mathcal{H} \subset \mathcal{G}$ is a closed supergroup subscheme and $\Psi: \mathcal{H} \times \mathcal{H} \rightarrow \mathbb{G}_{m}$ is a normalized even 2-cocycle.

Example 6.13. Let $V$ be a one-dimensional odd vector space, and consider the purely odd finite supergroup scheme $\mathcal{G}:=V$. By Example 5.7 and Theorem 6.12, the tensor category $\operatorname{sep}(V)$ has exactly six nonequivalent indecomposable exact left module categories corresponding to the pairs $(0,0),(V, 0)$ and $(V, B)$, where $B(v, v)=1$. Namely, the categories $\mathcal{N}(0,0)=$ sVect, $\mathcal{N}^{\circ}(0,0)=\operatorname{Vect}, \mathcal{N}(V, 0)=\operatorname{sMod}(\wedge V)$, $\mathcal{N}^{\circ}(V, 0)=\operatorname{Mod}(\wedge V), \mathcal{N}(V, B)=\operatorname{sMod}\left(k \mathbb{Z}_{2}\right)=$ Vect (here $k(\mathbb{Z} / 2 \mathbb{Z})$ is viewed as a superalgebra, where the generator of $\mathbb{Z} / 2 \mathbb{Z}$ is odd), and $\mathcal{N}^{\circ}(V, B)=\operatorname{Mod}(\mathbb{Z} / 2 \mathbb{Z})=$ sVect.

\section{The Classification of triangular Hopf algebras With The Chevalley PROPERTy}

In Sections 7.1, 7.2 we assume that $\mathcal{G}$ is a finite supergroup scheme over $k$. (The even case is treated in [G2, Sections 6.1-6.3].)

7.1. Twists for $k \mathcal{G}$. By AEGN, Theorem 5.7], there is a bijection between non-degenerate twists for $k \mathcal{G}$ and non-degenerate twists for $\mathcal{O}(\mathcal{G})$. Hence, as a consequence of Theorem [6.12, we deduce the following strengthening of Corollary 6.10.

Corollary 7.1. Let $\mathcal{G}$ be a finite supergroup scheme over $k$. The following four sets are in canonical bijection with each other:

(1) Equivalence classes of tensor structures on the forgetful functor $\operatorname{sRep}(\mathcal{G}) \rightarrow$ Vect

(2) Gauge equivalence classes of twists for $k \mathcal{G}$.

(3) Conjugacy classes of pairs $(\mathcal{H}, \Psi)$, where $\mathcal{H} \subset \mathcal{G}$ is a closed supergroup subscheme and $\Psi: \mathcal{H} \times \mathcal{H} \rightarrow \mathbb{G}_{m}$ is a non-degenerate even 2-cocycle. 
(4) Conjugacy classes of pairs $(\mathcal{H}, \mathcal{J})$, where $\mathcal{H} \subset \mathcal{G}$ is a closed supergroup subscheme and $\mathcal{J}$ is a non-degenerate twist for $k \mathcal{H}$.

Remark 7.2. If moreover, $\left[\mathfrak{g}_{1}, \mathfrak{g}_{1}\right]=0$ (e.g., in characteristic 0 , or if $\left.k \mathcal{G}=k \mathcal{G}_{0} \ltimes \wedge \mathfrak{g}_{1}\right)$, then each one of the above four sets is in bijection with the set of conjugacy classes of quadruples $\left(\mathcal{H}_{0}, \psi, \mathfrak{h}_{1}, B\right)$, where $\mathcal{H}_{0} \subset \mathcal{G}_{0}$ is a closed subgroup scheme, $\psi: \mathcal{H}_{0} \times \mathcal{H}_{0} \rightarrow k$ is a non-degenerate 2-cocycle, $Y \subset \mathfrak{g}_{1}$ is an $\mathcal{H}_{0}$-invariant subspace, and $B \in S^{2} \mathfrak{h}_{1}^{*}$ is nondegenerate (see 2.1).

Remark 7.3. Corollary 7.1 was proved for étale group schemes in [Mo, EG1, AEGN], for finite supergroups $\mathcal{G}$ such that $k \mathcal{G}=k \mathcal{G}_{0} \ltimes \wedge \mathfrak{g}_{1}$ in [EO], and for finite group schemes in G2].

Example 7.4. Retain the notation from Example 6.13. Then the tensor category $\operatorname{sRep}(V)$ has exactly two non-equivalent fiber functors to Vect corresponding to the left module categories $\mathcal{N}^{\circ}(0,0)$ and $\mathcal{N}(V, B)$.

7.2. Minimal twists for $k \mathcal{G}$. Recall that a twist $\mathcal{J}$ for $k \mathcal{G}$ is called minimal if the triangular Hopf superalgebra $\left((k \mathcal{G})^{\mathcal{J}}, \mathcal{J}_{21}^{-1} \mathcal{J}\right)$ is minimal, i.e., if the left (right) tensorands of $\mathcal{J}_{21}^{-1} \mathcal{J}$ span $k \mathcal{G}[\mathrm{R}]$.

By [G2, Proposition 6.7], a twist for a finite group scheme is minimal if and only if it is non-degenerate. In this section we extend this result to the super case, using the following result (see [EG2, Lemma A.8] and $[\mathrm{B}$, Proposition 1]).

Proposition 7.5. Let $\mathcal{D}$ and $\mathcal{E}$ be symmetric tensor categories over $k$, and suppose there exists a surjectiv 6 symmetric tensor functor $F$ : $\mathcal{D} \rightarrow \mathcal{E}$. If $\mathcal{D}$ is finitely tensor-generated and (super-)Tannakian, then so is $\mathcal{E}$.

We can now state and prove the first main result of this section.

Proposition 7.6. Let $\mathcal{G}$ be a finite supergroup scheme over $k$, and let $\mathcal{J}$ be a twist for $k \mathcal{G}$. Then $\mathcal{J}$ is minimal if and only if it is nondegenerate.

Proof. Suppose $\mathcal{J}$ is minimal. By Corollary 7.1, there exist a closed supergroup subscheme $\mathcal{H} \subset \mathcal{G}$ and a non-degenerate twist $\overline{\mathcal{J}}$ for $k \mathcal{H}$, such that the image of $\overline{\mathcal{J}}$ under the embedding $(k \mathcal{H})_{\mathcal{J}} \hookrightarrow(k \mathcal{G})_{\mathcal{J}}$ is $\mathcal{J}$. Since $\mathcal{J}$ is minimal and $\mathcal{H} \subset \mathcal{G}$, it follows that $\mathcal{H}=\mathcal{G}$.

Conversely, suppose $\mathcal{J}$ is non-degenerate. Let $\left(\mathcal{A}, \mathcal{J}_{21}^{-1} \mathcal{J}\right)$ be the minimal triangular Hopf sub-superalgebra of $\left((k \mathcal{G})^{\mathcal{J}}, \mathcal{J}_{21}^{-1} \mathcal{J}\right)$. The restriction functor $\operatorname{sRep}(\mathcal{G}) \rightarrow \operatorname{sRep}(\mathcal{A})$ is a surjective symmetric tensor

\footnotetext{
${ }^{6}$ I.e., any object $X \in \mathcal{E}$ is isomorphic to a subquotient of $F(V)$ for some $V \in \mathcal{D}$.
} 
functor. Thus by Proposition 7.5, $\operatorname{sRep}(\mathcal{A})$ is equivalent to $\operatorname{sep}(\mathcal{H}, u)$, as a symmetric tensor category, for some closed supergroup subscheme $\mathcal{H} \subset \mathcal{G}$. Now, it is a standard fact (see, e.g., G1]) that such an equivalence functor gives rise to a twist $\mathcal{I} \in(k \mathcal{H})^{\otimes 2}$ and an isomorphism of triangular Hopf superalgebras $\left((k \mathcal{H})^{\mathcal{I}}, \mathcal{I}_{21}^{-1} \mathcal{I}\right) \cong\left(\mathcal{A}, \mathcal{J}_{21}^{-1} \mathcal{J}\right)$.

We therefore get an injective homomorphism of triangular Hopf superalgebras $\left((k \mathcal{H})^{\mathcal{I}}, \mathcal{I}_{21}^{-1} \mathcal{I}\right) \hookrightarrow\left((k \mathcal{G})^{\mathcal{J}}, \mathcal{J}_{21}^{-1} \mathcal{J}\right)$, which implies that $\mathcal{J I}^{-1}$ is a symmetric twist for $k \mathcal{G}$. But by [DM, Theorem 3.2], this implies that $\mathcal{J I}^{-1}$ is gauge equivalent to $1 \otimes 1$. Therefore, the triangular Hopf superalgebras $\left((k \mathcal{G})^{\mathcal{J I}^{-1}}, \mathcal{I}_{21} \mathcal{J}_{21}^{-1} \mathcal{J I}^{-1}\right)$ and $(k \mathcal{G}, 1 \otimes 1)$ are isomorphic. In other words, $\left((k \mathcal{G})^{\mathcal{I}}, \mathcal{I}_{21}^{-1} \mathcal{I}\right)$ and $\left((k \mathcal{G})^{\mathcal{J}}, \mathcal{J}_{21}^{-1} \mathcal{J}\right)$ are isomorphic as triangular Hopf superalgebras, i.e., the pairs $(\mathcal{G}, \mathcal{J})$ and $(\mathcal{H}, \mathcal{I})$ are conjugate. We thus conclude from Corollary 7.1 that $\mathcal{H}=\mathcal{G}$, and hence that $\mathcal{J}$ is a minimal twist, as required.

Remark 7.7. Corollary 7.1 and Proposition 7.6 extend [G2, Corollary $6.3 \&$ Proposition 6.7] to the super case.

7.3. Triangular Hopf algebras. Let $(H, R)$ be a finite dimensional triangular Hopf algebra with the Chevalley property over $k$. Recall that by [EG2, Corollary 4.1], $(H, R)$ is twist equivalent to a finite dimensional triangular Hopf algebra with $R$-matrix of rank $\leq 2$ (i.e., to a modified supergroup algebra [AEG, Definition 3.3.4]). Hence by AEG, Corollary 3.3.3], $(H, R)$ corresponds to a unique pair $(\mathcal{G}, \epsilon)$, where $\mathcal{G}$ is a finite supergroup scheme over $k$ (see 2.1). Thus Corollary $7.1 \mathrm{im}$ plies the following classification result, which extends [EG2, Theorem 5.1] to arbitrary finite dimensional triangular Hopf algebras with the Chevalley property over $k$.

Theorem 7.8. The following three sets are in canonical bijection with each other:

(1) Isomorphism classes of finite dimensional triangular Hopf algebras $(H, R)$ with the Chevalley property over $k$.

(2) Conjugacy classes of quadruples $(\mathcal{G}, \mathcal{H}, \mathcal{J}, \epsilon)$, where $\mathcal{G}$ is a finite supergroup scheme over $k, \mathcal{H} \subset \mathcal{G}$ is a closed supergroup subscheme, $\mathcal{J}$ is a minimal twist for $k \mathcal{H}$, and $\epsilon \in \mathcal{G}(k)$ is a central element of order $\leq 2$ acting by -1 on $\mathfrak{g}_{1}$.

(3) Conjugacy classes of quadruples $(\mathcal{G}, \mathcal{H}, \Psi, \epsilon)$, where $\mathcal{G}$ is a finite supergroup scheme over $k, \mathcal{H} \subset \mathcal{G}$ is a closed supergroup subscheme, $\Psi$ is a non-degenerate even 2 -cocycle on $\mathcal{H}$ with coefficients in $\mathbb{G}_{m}$, and $\epsilon \in \mathcal{G}(k)$ is a central element of order $\leq 2$ acting by -1 on $\mathfrak{g}_{1}$. 
Remark 7.9. The correspondence between (1) and (2) in Theorem 7.8 is given by $(H, R)=\overline{\left((k \mathcal{G})^{\mathcal{J}}, \epsilon\right)}$ (see [AEG, Theorem 3.3.1]; see also 3). A 2-cocycle $\Psi$ on $\mathcal{H}$ as in Theorem $7.8(3)$ determines a module category over $\operatorname{sRep}(\mathcal{G})$ of rank 1, i.e., a tensor structure on the forgetful functor $\operatorname{sRep}(\mathcal{G}) \rightarrow$ Vect, thus a twist $\mathcal{J}$ for $k \mathcal{G}$ supported on $\mathcal{H}$.

\section{REFERENCES}

[AEG] N. Andruskiewitch, P. Etingof and S. Gelaki. Triangular Hopf algebras with the Chevalley property. Michigan Journal of Mathematics 49 (2001), $277-298$.

[AEGN] E. Aljadeff, P. Etingof, S. Gelaki and D. Nikshych. On twisting of finitedimensional Hopf algebras. Journal of Algebra 256 (2002), 484-501.

[B] R. Bezrukavnikov. On tensor categories attached to cells in affine Weyl groups. Representation theory of algebraic groups and quantum groups, 69-90, Adv. Stud. Pure Math., 40, Math. Soc. Japan, Tokyo, 2004.

[D] P. Deligne. Categories Tannakiennes. In The Grothendick Festschrift, Vol. II, Prog. Math. 87 (1990), 111-195.

[DM] P. Deligne and J. Milne. Tannakian Categories. Lecture Notes in Mathematics 900, 101-228, 1982.

[EG1] P. Etingof and S. Gelaki. The classification of triangular semisimple and cosemisimple Hopf algebras over an algebraically closed field. International Mathematics Research Notices (2000), no. 5, 223-234.

[EG2] P. Etingof and S. Gelaki. Finite symmetric integral tensor categories with the Chevalley property, with an Appendix by Kevin Coulembier and Pavel Etingof. International Mathematics Research Notices, rnz093, https://doi.org/10.1093/imrn/rnz093.

[EO] P. Etingof and V. Ostrik. Finite tensor categories. Mosc. Math. J. 4 (2004), no. 3, 627-654, 782-783.

[EGNO] P. Etingof, S. Gelaki, D. Nikshych and V. Ostrik. Tensor Categories. AMS Mathematical Surveys and Monographs book series 205 (2015), 362 pp.

[G1] S. Gelaki. On the classification of finite-dimensional triangular Hopf algebras. New directions in Hopf algebras, 69-116, Math. Sci. Res. Inst. Publ., 43 (2002), Cambridge Univ. Press, Cambridge.

[G2] S. Gelaki. Module categories over affine group schemes. Quantum Topology 6 (2015), no. 1, 1-37.

[Ma] A. Masuoka. The fundamental correspondences in super affine groups and super formal groups. J. Pure Appl. Algebra 202 (2005), 284-312.

[Mo] M. Movshev. Twisting in group algebras of finite groups. Func. Anal. Appl. 27 (1994), 240-244.

[MS] A. Masuoka and T. Shibata. On functor points of affine supergroups.

[R] D.E. Radford. Minimal quasitriangular Hopf algebras. Journal of Algebra 157 (1993), 285-315.

[W] W. Waterhouse. Introduction to affine group schemes. Graduate Texts in Mathematics, 66. Springer-Verlag, New York-Berlin, 1979. xi+164 pp.

Department of Mathematics, Iowa State University, Ames IA, USA

Email address: gelaki@iastate.edu 